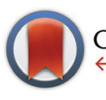

CrossMark \&lick for updates

Cite this: Polym. Chem., 2015, 6 , 5705

\section{The synthesis and aqueous solution properties of sulfobutylbetaine (co)polymers: comparison of synthetic routes and tuneable upper critical solution temperatures $\uparrow$}

\author{
Yicheng Zhu, Janina-Miriam Noy, $\$$ Andrew B. Lowe $\$$ and Peter J. Roth $\$$
}

Polysulfobutylbetaine (SBB) (co)polymers, zwitterionic species bearing ammonium and sulfonate groups separated by a butyl spacer in every repeat unit, were prepared through three different synthetic routes and their aqueous solution behaviour was studied. Postpolymerization quaternization of poly[2-(dimethylamino)ethyl methacrylate] with 1,4-butanesultone resulted in incomplete modification due to the low reactivity of this alkylating agent. RAFT radical polymerization of SBB-functional (meth)acrylate monomers and their copolymerization with a sulfopropylbetaine (SPB) methacrylate yielded well-defined (co)polymers with low dispersities $1.13 \leq \emptyset_{M} \leq 1.23$ at monomer conversions of $75-92 \%$. For a series of SBB methacrylate homopolymers with increasing degrees of polymerization from 66-186 measured upper critical solution temperature (UCST) cloud points increased from $27-77^{\circ} \mathrm{C}$. Cloud points of statistical SPB-SBB copolymers with similar degrees of polymerization, but varying molar compositions, increased linearly with SBB content offering a simple means of UCST tuning. Additionally, novel SBB acrylamide homo- and copolymers were prepared by postpolymerization modification of poly(pentafluorophenyl acrylate) with an SBB-functional amine and in mixtures with benzylamine as a hydrophobic modifier. In all cases, the SBB (co)polymers had significantly higher UCSTs than their more common SPB counterparts, greatly extending the temperature range of tuneable UCST transitions and making the investigated SBB (co)polymers advantageous for exploiting their 'smart' behaviour. In this respect, combining SBB functionality with hydrophobic benzylacrylamide comonomers is presented as a simple means of increasing the maximum salt concentration at which UCST behaviour (which shows an antipolyelectrolyte effect) can be observed, enabling UCST transitions in aqueous solutions containing a physiological concentration $(9 \mathrm{~g}$ $\mathrm{L}^{-1}$ ) of $\mathrm{NaCl}$.
Received 3rd February 2015, Accepted 6th April 2015 DOI: $10.1039 / c 5 p y 00160 a$ www.rsc.org/polymers

\section{Introduction}

Thermoresponsive polymers have been the focus of research for many decades and a range of applications including drug delivery, separation, diagnostics, and tissue engineering are based on polymers exhibiting aqueous inverse temperature solubility (lower critical solution temperature (LCST) behaviour, i.e. phase separation above a critical temperature). ${ }^{1}$ Their

Centre for Advanced Macromolecular Design (CAMD), School of Chemical Engineering, University of New South Wales, Kensington, Sydney, NSW 2052, Australia.E-mail: Peter.Roth@curtin.edu.au

$\dagger$ Electronic supplementary information (ESI) available: Heating and cooling curves showing reproducibility and hysteresis, ${ }^{19} \mathrm{~F}$ NMR measurements indicating full conversion of PFP precursors. See DOI: 10.1039/c5py00160a

$\$$ Present address: Nanochemistry Research Institute (NRI), Department of Chemistry, Curtin University, Bentley, Perth WA 6102, Australia. counterparts, polymers with an aqueous upper critical solution temperature (UCST) which phase separate below a critical temperature, have similar potential in such applications, but only a few (co)polymers are known to exhibit this type of "smart" behaviour. ${ }^{2}$ Sulfobetaine polymers, ${ }^{3-6}$ which carry permanently ( $\mathrm{pH}$ independent) charged ammonium and sulfonate groups in every repeat unit, are promising candidates for aqueous UCST behaviour because their zwitterionic side groups can cause strong inter- and intrapolymer attractions through electrostatic interlocking at low temperatures resulting in insolubility. Polysulfobetaines have additionally attracted attention because of their superior haemocompatibility and antibiofouling properties, ${ }^{7,8}$ which have been exploited for surface modification of ultrafiltration membranes ${ }^{9}$ and blood-contacting devices ${ }^{10,11}$ and wound dressing applications. ${ }^{12}$ Since first described in the $1950 \mathrm{~s},{ }^{13}$ a wide range of polysulfobetaines with various backbone types, side group geometries, ammonium alkyl substituents, and spacer lengths 
has been prepared. ${ }^{3,4,6,14-18}$ Notably, a significant focus of previous research efforts was on fully water soluble (co)polymers with miscibility gaps often being considered a nuisance rather than an opportunity. Consequently, aqueous UCST behaviour has only been reported for a small subset of zwitterionic (co)polymers. ${ }^{3,19-25}$ While the influence of spacer lengths, including the length of the ion bridge between the charged sites, on hydrophilicity has been investigated for small molecule (sulfo)betaines, ${ }^{15,26-29}$ detailed studies of aqueous solution behaviour of sulfobetaine polymers have invariably dealt with sulfopropylbetaine (SPB) polymers, i.e. structures with three methylene groups between the charged groups. ${ }^{19-21,23,24,30-32}$ The majority of recent studies on polysulfobetaine UCST behaviour is, in fact, largely based on the two commercially available monomers 3-((2-(methacryloyloxy)ethyl)dimethylammonio)propane-1-sulfonate (MA2-3) 21,30,33-35 and 3-((3-methacrylamidopropyl)dimethylammonio)propane1-sulfonate (MAm3-3). ${ }^{21,31,36,37}$

Zwitterionic monomers are typically polymerized in aqueous solution, ${ }^{3,16,38}$ which poses limitations for the incorporation of hydrophobic segments including co-monomers. ${ }^{39}$ Also, characterization of zwitterionic polymers, especially by size exclusion chromatography (SEC), suffers from the limited solubility of such polymers in organic solvents. ${ }^{32}$ For these reasons, quaternization of amino groups or installation of zwitterionic segments through postpolymerization modification of (easier to characterize) precursors can be preferential. ${ }^{6}$ Commonly, tertiary amine functional polymers, such as homo- $^{16,40}$ or copolymers ${ }^{41,42}$ of 2-(dimethylamino)ethyl methacrylate (DMAEMA) are reacted with sultones, cyclic sulfonic esters, in organic solvents producing, in the case of pDMAEMA and 1,3-propanesultone, poly[3-((2-(methacryloyloxy)ethyl)dimethylammonio)propane-1-sulfonate], p(MA2-3). For reaction in most organic solvents, such as THF, the resulting partially betainized (co)polymers precipitate with reaction continuing, considerably slower, ${ }^{16}$ under heterogeneous conditions. We recently reported ${ }^{43}$ the postmodification of the activated ester precursor poly(pentafluorophenyl acrylate), pPFPA $^{44}$ with a zwitterionic functional primary amine, 3-((3-aminopropyl)dimethylammonio)propane-1-sulfonate, amine33 , as a novel and versatile synthetic route toward zwitterionic homo- and hydrophobically modified copolymers. Notably, also in this study only propyl-spaced derivatives were investigated.

Though promising, applications of the UCST behaviour of polysulfobetaines are limited compared to those of LCST-type polymers such as poly( $N$-isopropyl acrylamide) or non-linear poly(ethylene glycol)s for two reasons. Firstly, UCST transitions generally show a strong positive dependence on molecular weight. As the commonly studied MA2-3 and MAm3-3 based polysulfopropyl species can exhibit good solubility in pure water, relatively high molecular weights of several hundred $\mathrm{kg}$ $\mathrm{mol}^{-1}$ are necessary to achieve cloud points as high as 30-40 ${ }^{\circ} \mathrm{C}^{21,35}$ Polymers with lower molecular weights have lower critical temperatures or may be fully soluble, not exhibiting the desired "smart" behaviour at all. Secondly, zwitterionic polymers show an antipolyelectrolyte effect: added salts screen the inter- and intraionic interactions, reducing electrostatic interlocking efficiency which results in a decrease of UCST cloud points and, ultimately, at sufficiently high salt concentrations, in temperature independent aqueous solubility. ${ }^{4}$ Consequently, it is of considerable interest to develop (co)polymers with significantly higher UCST transitions than those of comparable MA2-3 and MAm3-3 based polysulfopropylbetainespossibly to an extent that polymers are insoluble in pure water over the entire temperature range up to $100{ }^{\circ} \mathrm{C}$ at $1 \mathrm{~atm}$. "Smart" behaviour in a practical temperature range would then be observable for (more conveniently available) lower molecular weight samples, and, importantly, in aqueous salt solutions, most ideally extending to a physiologically relevant ion concentration (e.g. $\sim 154 \mathrm{mM} \mathrm{NaCl}$ ). Our previously reported postpolymerization preparation of hydrophobically modified sulfopropylbetaine copolymers represents a first study aimed at increasing UCST transitions through tuning of copolymer composition which enabled sharp transitions of a copolymer with $M_{\mathrm{n}}=27 \mathrm{~kg} \mathrm{~mol}{ }^{-1}$ in aqueous solutions containing up to $76 \mathrm{mM} \mathrm{NaCl}{ }^{43}$

Herein, we present a detailed study into the UCST behaviour of sulfobutylbetaine (SBB) (co)polymers and show that increasing the ion bridge by just one methylene unit represents a simple means of significantly increasing critical solution temperatures of homopolymers. SBB (co)polymers and several SPB reference samples were prepared through direct (co)polymerization of commercial and prepared zwitterionic monomers, through postmodification of pDMAEMA with sultones, and through postmodification of an activated ester precursor using a novel zwitterionic amine allowing us to compare these methods with regards to their efficiency in producing the SBB species and providing access to a library of (novel) zwitterionic acrylate, methacrylate and (hydrophobically modified) acrylamide (co)polymers with tuneable UCST transitions spanning an impressive temperature range and extending up to physiologically relevant $\mathrm{NaCl}$ concentrations.

\section{Experimental section}

\section{Materials}

All reagents, including methacryloxyethyl dimethylammonio propanesulfonate (MA2-3) and 2-cyano-2-propyl dithiobenzoate (CPDB) were purchased from Sigma-Aldrich and were used as received unless stated otherwise. Propylene carbonate (99.7\%, anhydrous) was stored in a glovebox. Azobis(isobutyronitrile) (AIBN) was recrystallized from methanol and stored at $-24^{\circ} \mathrm{C}$. The syntheses of the chain transfer agent (CTA) benzyl propyl trithiocarbonate (BPTC), ${ }^{45}$ the CTA pentafluorophenyl 4-cyano4 -((phenylcarbonothioyl)thio)valerate, ${ }^{46}$ the activated ester initiator bis(pentafluorophenyl) 4,4'-(diazene-1,2-diyl)bis(4-cyanopentanoate), ${ }^{46}$ and the amine-functional dye 4-nitro7-piperazin-1-yl-2,1,3-benzoxadiazole (NBD amine) ${ }^{47}$ are described elsewhere. 


\section{Methods}

NMR spectroscopic measurements in $\mathrm{D}_{2} \mathrm{O}$ were performed on a Bruker Avance $300 \mathrm{MHz}$ instrument in $5 \mathrm{~mm}$ NMR tubes. Measurements of polymers were done on $\mathrm{D}_{2} \mathrm{O}$ solutions containing up to $0.5 \mathrm{M} \mathrm{NaCl}$. The internal solvent signal $\delta\left(\mathrm{D}_{2} \mathrm{O}\right)=$ $4.79 \mathrm{ppm}$ was used as reference.

Size exclusion chromatography (SEC) in $N, N$-dimethylacetamide (DMAc) was performed on a Shimadzu system with four $300 \times 7.8 \mathrm{~mm}^{2}$ linear phenogel columns $\left(10^{5}, 10^{4}, 10^{3}\right.$, and $500 \AA$ ) operating at a flow rate of $1 \mathrm{~mL} \mathrm{~min}{ }^{-1}$. The system was calibrated with a series of low dispersity polystyrene (PS) standards with molar masses ranging from $0.58-1820 \mathrm{~kg} \mathrm{~mol}^{-1}$. Aqueous SEC was performed on a Shimadzu system with two Agilent Aquagel columns with $0.2 \mathrm{M} \mathrm{NaCl}$ solution containing 0.02 mass $\%$ sodium azide as eluent at a flow rate of $1 \mathrm{~mL}$ $\min ^{-1}$. This system was calibrated with a series of narrow molar mass distribution poly(ethylene glycol) (PEG) standards. Chromatograms were analysed by Cirrus SEC software version 3.0 .

Fourier transform infrared spectroscopy (FT-IR) was performed on a Bruker IFS 66/S instrument under attenuated total reflectance, and data was analysed with OPUS software version 4.0 .

Turbidity measurements were performed on a Varian Cary 300 Scan spectrophotometer equipped with a Cary temperature controller and a Peltier heating element in quartz cuvettes of $10 \mathrm{~mm}$ path length at a wavelength of $520 \mathrm{~nm}$ with heating/ cooling rates of $1{ }^{\circ} \mathrm{C} \mathrm{min}^{-1}$. Unless otherwise noted, polymer concentrations were $10 \mathrm{~g} \mathrm{~L}^{-1}$. For clear solutions the baseline was corrected to zero absorbance, $A$. Transmittance, $t=10^{-A}$, was plotted against temperature, and cloud points, CP, were determined at the onset of transmittance decrease.

Electrospray ionization (ESI) mass spectrometry was performed on a Scientific LTQ Orbitrap XL mass spectrometer operating in positive ion mode with a spray voltage of $1.2 \mathrm{kV}$, a capillary voltage of $45 \mathrm{~V}$, a capillary temperature of $200{ }^{\circ} \mathrm{C}$, and a tube lens voltage of $120 \mathrm{~V}$.

Poly[2-(dimethylamino)ethyl methacrylate], pDMAEMA, was prepared as previously described. ${ }^{48} M_{\mathrm{n}}^{\text {theor. }}=31.8 \mathrm{~kg} \mathrm{~mol}^{-1}$, $\mathrm{DP}^{\text {theor. }}=201, M_{\mathrm{n}}^{\mathrm{SEC}}=22.3 \mathrm{~kg} \mathrm{~mol}^{-1}$ (DMAc, PS standard), $\emptyset_{\mathrm{M}}=M_{\mathrm{w}}^{\mathrm{SEC}} / M_{\mathrm{n}}^{\mathrm{SEC}}=1.14 .{ }^{1} \mathrm{H} \mathrm{NMR}\left(\mathrm{D}_{2} \mathrm{O}, 300 \mathrm{MHz}\right), \delta / \mathrm{ppm}=4.11$ $\left(-\mathrm{OCH}_{2} \mathrm{CH}_{2}-\right), 2.67\left(-\mathrm{OCH}_{2} \mathrm{CH}_{2}-\right), 2.27,2.04$ (backbone $-\mathrm{CH}_{2}{ }^{-}$), 1.08, 0.90 (backbone $-\mathrm{CH}_{3}$ ).

Postpolymerization of pDMAEMA with sultones. Poly[3-((2(methacryloyloxy)ethyl)dimethylammonio)propane-1-sulfonate], p(MA2-3): pDMAEMA (100 mg, $0.636 \mathrm{mmol}$ of repeat units, 1 eq.) was dissolved in 2,2,2-trifluoroethanol ( $2 \mathrm{~mL})$. In a separate vial, 1,3-propanesultone ( $155.4 \mathrm{mg}, 1.272 \mathrm{mmol}, 2$ eq.) was dissolved in TFE $(1 \mathrm{~mL})$ and then added into the polymer solution. The mixture was stirred at $40{ }^{\circ} \mathrm{C}$ for 3 days. Brine was added to the reaction mixture and the aqueous phase was washed several times with diethyl ether, subjected to dialysis utilizing regenerated cellulose membranes with a $3500 \mathrm{~g} \mathrm{~mol}^{-1}$ molecular weight cut-off in ultrapure water for 3 days followed by freeze drying, yielding $163 \mathrm{mg}$ (92\%) of a white solid.
${ }^{1} \mathrm{H}$ NMR $\left(\mathrm{D}_{2} \mathrm{O}, 500 \mathrm{mM} \mathrm{NaCl}, 300 \mathrm{MHz}\right), \delta / \mathrm{ppm}=4.54$ $\left(-\mathrm{OCH}_{2} \mathrm{CH}_{2}-\right), 3.86\left(-\mathrm{OCH}_{2} \mathrm{CH}_{2}{ }^{-}\right), 3.65\left(-\mathrm{N}^{+}\left(\mathrm{CH}_{3}\right)_{2} \mathrm{CH}_{2} \mathrm{CH}_{2}^{-}\right)$, $3.28\left(-\mathrm{N}^{+}\left(\mathrm{CH}_{3}\right)_{2}-\right), 3.03\left(-\mathrm{CH}_{2} \mathrm{SO}_{3}{ }^{-}\right), 2.34\left(-\mathrm{N}^{+}\left(\mathrm{CH}_{3}\right)_{2} \mathrm{CH}_{2} \mathrm{CH}_{2}{ }^{-}\right)$, 2.06 (backbone $-\mathrm{CH}_{2}-$ ), 1.20, 1.06 (backbone $-\mathrm{CH}_{3}$ ).

Poly[4-((2-(methacryloyloxy)ethyl)dimethylammonio)butane1-sulfonate], p(MA2-4), was prepared in an analogous procedure using 1,4-butanesultone (173.2 mg, $1.272 \mathrm{mmol}, 2$ eq.) with heating to reflux (oil bath at $103{ }^{\circ} \mathrm{C}$ ) for 4 days. Workup as detailed above yielded $125 \mathrm{mg}(67 \%)$ of a white solid. ${ }^{1} \mathrm{H} \mathrm{NMR}\left(\mathrm{D}_{2} \mathrm{O}, 500 \mathrm{mM} \mathrm{NaCl}, 300 \mathrm{MHz}\right), \delta / \mathrm{ppm}=4.57$ $\left(-\mathrm{OCH}_{2} \mathrm{CH}_{2}-\right), 3.86\left(-\mathrm{OCH}_{2} \mathrm{CH}_{2}^{-}\right), 3.56\left(-\mathrm{N}^{+}\left(\mathrm{CH}_{3}\right)_{2} \mathrm{CH}_{2} \mathrm{CH}_{2} \mathrm{CH}_{2}-\right)$, $3.28\left(-\mathrm{N}^{+}\left(\mathrm{CH}_{3}\right)_{2}{ }^{-}\right), \quad 3.03 \quad\left(-\mathrm{CH}_{2} \mathrm{SO}_{3}{ }^{-}\right), \quad 2.08 \quad\left(-\mathrm{N}^{+}\left(\mathrm{CH}_{3}\right)_{2} \mathrm{CH}_{2}{ }^{-}\right.$ $\mathrm{CH}_{2} \mathrm{CH}_{2}-$ and backbone $\left.-\mathrm{CH}_{2}-\right), 1.90\left(-\mathrm{N}^{+}\left(\mathrm{CH}_{3}\right)_{2} \mathrm{CH}_{2} \mathrm{CH}_{2} \mathrm{CH}_{2}^{-}\right)$, 1.20, 1.06 (backbone $-\mathrm{CH}_{3}$ ).

Monomer 4-((2-(methacryloyloxy)ethyl)dimethylammonio)butane-1-sulfonate (MA2-4). ${ }^{49}$ 2-(Dimethylamino)ethyl methacrylate (DMAEMA, $4.43 \mathrm{~mL}, 26.3 \mathrm{mmol}$ ), 1,4-butanesultone (2.44 mL, $23.9 \mathrm{mmol}$ ), inhibitor 3,5-di-tert-butyl-4-hydroxytoluene (BHT, $50 \mathrm{mg})$, and acetonitrile $(50 \mathrm{~mL})$ were combined and refluxed for $48 \mathrm{~h}$. The consumption of 1,4-butanesultone was monitored by thin-layer chromatography (TLC) using $n$-hexane-ethyl acetate $50: 50$ as the mobile phase. The crude material which precipitated throughout the course of the reaction was filtered, washed with acetonitrile $(150 \mathrm{~mL})$, and dried in vacuo at room temperature. $5.32 \mathrm{~g}(76 \%)$ of a white solid were obtained. ${ }^{1} \mathrm{H}$ NMR $\left(\mathrm{D}_{2} \mathrm{O}, 300 \mathrm{MHz}\right), \delta / \mathrm{ppm}=6.18$ $\left(\mathrm{m}, 1 \mathrm{H}, \mathrm{CHH}=\mathrm{C}\left(\mathrm{CH}_{3}\right)^{-}\right.$, cis $), 5.80\left(\mathrm{~m}, 1 \mathrm{H}, \mathrm{CH}=\mathrm{C}\left(\mathrm{CH}_{3}\right)^{-}\right.$, trans), $4.65\left(\mathrm{~m}, 2 \mathrm{H},-\mathrm{OCH}_{2} \mathrm{CH}_{2}-\right), 3.80\left(\mathrm{~m}, 2 \mathrm{H},-\mathrm{OCH}_{2} \mathrm{CH}_{2}-\right)$, $3.48\left(\mathrm{~m}, 2 \mathrm{H}, \quad-\mathrm{N}^{+}\left(\mathrm{CH}_{3}\right)_{2} \mathrm{CH}_{2} \mathrm{CH}_{2} \mathrm{CH}_{2}-\right), 3.20(\mathrm{~s}, 6 \mathrm{H}$, $\left.-\mathrm{N}^{+}\left(\mathrm{CH}_{3}\right)_{2}{ }^{-}\right), 2.99\left(\mathrm{t}, 2 \mathrm{H},{ }_{-} \mathrm{CH}_{2} \mathrm{SO}_{3}{ }^{-}\right), 2.00(\mathrm{~m}, 5 \mathrm{H}$, $-\mathrm{N}^{+}\left(\mathrm{CH}_{3}\right)_{2} \mathrm{CH}_{2} \mathrm{CH}_{2} \mathrm{CH}_{2}-$ and $\left.-\mathrm{CH}_{2} \mathrm{C}\left(\mathrm{CH}_{3}\right)^{-}\right), 1.82(\mathrm{~m}, 2 \mathrm{H}$, $\left.-\mathrm{N}^{+}\left(\mathrm{CH}_{3}\right)_{2} \mathrm{CH}_{2} \mathrm{CH}_{2} \mathrm{CH}_{2}-\right)$.

Monomer 4-((2-(acryloyloxy)ethyl)dimethylammonio)butane1-sulfonate (A2-4). 2-(Dimethylamino)ethyl acrylate (DMAEA, $4.19 \mathrm{~mL}, 27.6 \mathrm{mmol})$, 1,4-butanesultone (2.56 mL, $25.1 \mathrm{mmol}$ ), BHT $(50 \mathrm{mg})$, and acetonitrile $(50 \mathrm{~mL})$ were combined and refluxed for $48 \mathrm{~h}$. The consumption of 1,4-butanesultone was monitored by TLC with $n$-hexane-ethyl acetate $50: 50$ as the mobile phase. The crude material which precipitated throughout the course of the reaction was filtered, washed with acetonitrile $(150 \mathrm{~mL})$ and dried in vacuo at room temperature. $6.20 \mathrm{~g}$ $(89 \%)$ of a white solid were obtained. ${ }^{1} \mathrm{H}$ NMR $\left(\mathrm{D}_{2} \mathrm{O}\right.$, $300 \mathrm{MHz}), \delta / \mathrm{ppm}=6.49\left(\mathrm{dd}, 1 \mathrm{H}\right.$, trans $,{ }^{2} J_{\text {gem }}=1.2 \mathrm{~Hz},{ }^{3} J_{\text {cis }}=$ $17.4 \mathrm{~Hz}, \mathrm{CH}=\mathrm{CH}-), 6.25\left(\mathrm{dd}, 1 \mathrm{H}\right.$, gem, ${ }^{3} J_{\text {trans }}=17.1 \mathrm{~Hz}$, $\left.{ }^{3} J_{\text {cis }}=17.1 \mathrm{~Hz}, \mathrm{CHH}=\mathrm{CH}-\right), 6.06\left(\mathrm{dd}, 1 \mathrm{H}, \mathrm{cis},{ }^{2} J_{\text {gem }}=0.9 \mathrm{~Hz}\right.$, $\left.{ }^{3} J_{\text {trans }}=10.5 \mathrm{~Hz}, \mathrm{CH} H=\mathrm{CH}-\right), 4.66\left(\mathrm{~m}, 2 \mathrm{H},-\mathrm{OCH}_{2} \mathrm{CH}_{2}-\right), 3.79$ $\left(\mathrm{m}, 2 \mathrm{H},-\mathrm{OCH}_{2} \mathrm{CH}_{2}-\right), 3.48\left(\mathrm{~m}, 2 \mathrm{H},-\mathrm{N}^{+}\left(\mathrm{CH}_{3}\right)_{2} \mathrm{CH}_{2} \mathrm{CH}_{2} \mathrm{CH}_{2}-\right)$, $3.20\left(\mathrm{~s}, 6 \mathrm{H},-\mathrm{N}^{+}\left(\mathrm{CH}_{3}\right)_{2}{ }^{-}\right), 2.99\left(\mathrm{t}, 2 \mathrm{H},-\mathrm{CH}_{2} \mathrm{SO}_{3}{ }^{-}\right), 2.00(\mathrm{~m}, 2 \mathrm{H}$, $\left.-\mathrm{N}^{+}\left(\mathrm{CH}_{3}\right)_{2} \mathrm{CH}_{2} \mathrm{CH}_{2} \mathrm{CH}_{2}-\right), 1.82\left(\mathrm{~m}, 2 \mathrm{H},-\mathrm{N}^{+}\left(\mathrm{CH}_{3}\right)_{2} \mathrm{CH}_{2} \mathrm{CH}_{2} \mathrm{CH}_{2}-\right)$.

Monomer 4-((3-methacrylamidopropyl)dimethylammonio)butane-1-sulfonate (MAm3-4). 3-(Dimethylamino)propyl methacrylamide (DMAPMAm, $3.05 \mathrm{~g}, 17.9 \mathrm{mmol}$ ), 1,4-butanesultone (1.67 mL, $16.3 \mathrm{mmol}$ ), BHT (50 mg), and acetonitrile $(50 \mathrm{~mL})$ were combined and heated to $45^{\circ} \mathrm{C}$ for 3 days. The crude material which precipitated throughout the course of the reaction was filtered, washed with acetonitrile $(150 \mathrm{~mL})$ 
and dried in vacuo at room temperature. $2.63 \mathrm{~g}(53 \%)$ of a white solid were obtained. ${ }^{1} \mathrm{H}$ NMR $\left(\mathrm{D}_{2} \mathrm{O}, 300 \mathrm{MHz}\right), \delta / \mathrm{ppm}=$ $5.73(\mathrm{~m}, 1 \mathrm{H}, \mathrm{C} H \mathrm{H}=\mathrm{C}<), 5.50(\mathrm{~m}, 1 \mathrm{H}, \mathrm{CH} H=\mathrm{C}<), 3.38$ $\left(\mathrm{m}, 6 \mathrm{H},-\mathrm{NHCH}_{2} \mathrm{CH}_{2} \mathrm{CH}_{2}-\mathrm{N}^{+}\left(\mathrm{CH}_{3}\right)_{2} \mathrm{CH}_{2}-\right), 3.09(\mathrm{~s}, 6 \mathrm{H}$, $\left.-\mathrm{N}^{+}\left(\mathrm{CH}_{3}\right)_{2}{ }^{-}\right), 2.98\left(\mathrm{t}, 2 \mathrm{H},{ }^{-} \mathrm{CH}_{2} \mathrm{SO}_{3}{ }^{-}\right), 2.06(\mathrm{~m}, 2 \mathrm{H}$, $\left.-\mathrm{NHCH}_{2} \mathrm{CH}_{2} \mathrm{CH}_{2}-\right), 1.95\left(\mathrm{~m}, 5 \mathrm{H},-\mathrm{N}^{+}\left(\mathrm{CH}_{3}\right)_{2} \mathrm{CH}_{2} \mathrm{CH}_{2} \mathrm{CH}_{2}-\right.$ and $\left.\mathrm{CH}_{2}=\mathrm{C}\left(\mathrm{CH}_{3}\right)^{-}\right), 1.81\left(\mathrm{~m}, 2 \mathrm{H},-\mathrm{N}^{+}\left(\mathrm{CH}_{3}\right)_{2} \mathrm{CH}_{2} \mathrm{CH}_{2} \mathrm{CH}_{2}-\right)$. MS (ESI) $m / z(\%)=329(100)[\mathrm{M}+\mathrm{Na}]^{+}, 635(72)[2 \mathrm{M}+\mathrm{Na}]^{+}, 941$ (29) $[3 \mathrm{M}+\mathrm{Na}]^{+}, 482(16)[3 \mathrm{M}+2 \mathrm{Na}]^{2+}, 788(11)[5 \mathrm{M}+2 \mathrm{Na}]^{2+}$; m.p. $117^{\circ} \mathrm{C}$.

RAFT (co)polymerization of zwitterionic methacrylate monomers (procedure A). Zwitterionic monomer (MA2-3 or MA2-4, 0.75 g, 130-300 equiv. as given below), RAFT agent 2-cyano-2-propyl benzodithioate (CPDB, 1 equiv.), initiator AIBN (0.1 equiv.) and 2,2,2-trifluoroethanol (4 mL) were combined in a flask and degased, polymerized, analysed and purified as described above. The methacrylic copolymers $\mathbf{p}\left[(\mathbf{M A 2}-3)_{x^{-}}\right.$ co-(MA2-4) $)_{y}$ ] were synthesized in analogy using a mixture of monomers MA2-3 and MA2-4 in predetermined molar feed ratios. After purification, molar compositions were determined by ${ }^{1} \mathrm{H}$ NMR spectroscopy by comparing signals at $\delta / \mathrm{ppm}=2.34$ ( $2 \mathrm{H}$ from propyl spacer of MA2-3 repeat units) with $\delta / \mathrm{ppm}=$ 2.20-1.50 (2 $\mathrm{H}$ from MA2-3 and $6 \mathrm{H}$ from MA2-4 overlapping).

Poly[3-((2-(methacryloyloxy)ethyl)dimethylammonio)propane1-sulfonate], p(MA2-3): ${ }^{1} \mathrm{H}$ NMR $\left(\mathrm{D}_{2} \mathrm{O}, 500 \mathrm{mM} \mathrm{NaCl}\right.$, $300 \mathrm{MHz}), \delta / \mathrm{ppm}=4.56\left(-\mathrm{OCH}_{2} \mathrm{CH}_{2}-\right), 3.88\left(-\mathrm{OCH}_{2} \mathrm{CH}_{2}-\right), 3.66$ $\left(-\mathrm{N}^{+}\left(\mathrm{CH}_{3}\right)_{2} \mathrm{CH}_{2} \mathrm{CH}_{2}{ }^{-}\right), 3.30\left(-\mathrm{N}^{+}\left(\mathrm{CH}_{3}\right)_{2}{ }^{-}\right), 3.04\left(-\mathrm{CH}_{2} \mathrm{SO}_{3}{ }^{-}\right), 2.34$ $\left(-\mathrm{N}^{+}\left(\mathrm{CH}_{3}\right)_{2} \mathrm{CH}_{2} \mathrm{CH}_{2}{ }^{-}\right), 2.07$ (backbone $\left.-\mathrm{CH}_{2}{ }^{-}\right), 1.21$, 1.06 (backbone $\left.-\mathrm{CH}_{3}\right)$.

Poly[4-((2-(methacryloyloxy)ethyl)dimethylammonio)butane1-sulfonate], p(MA2-4): ${ }^{1} \mathrm{H}$ NMR $\left(\mathrm{D}_{2} \mathrm{O}, 500 \mathrm{mM} \mathrm{NaCl}\right.$, $300 \mathrm{MHz}), \delta / \mathrm{ppm}=4.56\left(-\mathrm{OCH}_{2} \mathrm{CH}_{2}-\right), 3.85\left(-\mathrm{OCH}_{2} \mathrm{CH}_{2}-\right), 3.54$ $\left(-\mathrm{N}^{+}\left(\mathrm{CH}_{3}\right)_{2} \mathrm{CH}_{2} \mathrm{CH}_{2} \mathrm{CH}_{2}-\right), 3.27\left(-\mathrm{N}^{+}\left(\mathrm{CH}_{3}\right)_{2}-\right), 3.02\left(-\mathrm{CH}_{2} \mathrm{SO}_{3}{ }^{-}\right)$, $2.06\left(-\mathrm{N}^{+}\left(\mathrm{CH}_{3}\right)_{2} \mathrm{CH}_{2} \mathrm{CH}_{2} \mathrm{CH}_{2}-\right.$ and backbone $\left.-\mathrm{CH}_{2}-\right), \quad 1.90$ $\left(-\mathrm{N}^{+}\left(\mathrm{CH}_{3}\right)_{2} \mathrm{CH}_{2} \mathrm{CH}_{2} \mathrm{CH}_{2}-\right), 1.20,1.06$ (backbone $-\mathrm{CH}_{3}$ ).

RAFT polymerization of zwitterionic acrylate monomer (procedure B). Zwitterionic monomer 4-((2-(acryloyloxy)ethyl)dimethylammonio)butane-1-sulfonate (A2-4) (0.75 g, 2.68 mmol, 200 equiv.), RAFT agent benzyl propyl trithiocarbonate (BPTC, $3.3 \mathrm{mg}, 0.0134 \mathrm{mmol}, 1$ equiv.), initiator AIBN (0.2 mg, $13.4 \mu \mathrm{mol}, 0.1$ equiv.) and solvent 2,2,2-trifluoroethanol ( $4 \mathrm{~mL}$ ) were combined in a flask equipped with a stir bar. The mixture was sealed with a rubber septum and purged with nitrogen for $20 \mathrm{~min}$ before being placed into a preheated oil bath at $70{ }^{\circ} \mathrm{C}$ overnight. Polymerization was stopped by quenching the reaction with liquid nitrogen. A sample $(50 \mu \mathrm{L})$ was withdrawn, diluted with $\mathrm{D}_{2} \mathrm{O}(600 \mu \mathrm{L})$ and analysed by ${ }^{1} \mathrm{H}$ NMR spectroscopy to determine monomer conversion through quantification of residual vinyl signals and comparison with polymeric signals. The product was purified by addition of brine and washing with diethyl ether, followed by dialysis in ultrapure water for 3 days and freeze-drying. Conversion and SEC-measured $M_{\mathrm{n}}^{\mathrm{SEC}}$ and $\bigoplus_{\mathrm{M}}$ are given below. Poly[4-((2(acryloyloxy)ethyl)dimethylammonio)butane-1-sulfonate], p(A2-4): ${ }^{1} \mathrm{H}$ NMR $\left(\mathrm{D}_{2} \mathrm{O}, 500 \mathrm{mM} \mathrm{NaCl}, 300 \mathrm{MHz}\right), \delta / \mathrm{ppm}=4.57$ $\left(-\mathrm{OCH}_{2} \mathrm{CH}_{2}-\right), 3.80\left(-\mathrm{OCH}_{2} \mathrm{CH}_{2}^{-}\right), 3.51\left(-\mathrm{N}^{+}\left(\mathrm{CH}_{3}\right)_{2} \mathrm{CH}_{2} \mathrm{CH}_{2} \mathrm{CH}_{2}-\right)$,
$3.24\left(-\mathrm{N}^{+}\left(\mathrm{CH}_{3}\right)_{2}{ }^{-}\right), 2.99\left(-\mathrm{CH}_{2} \mathrm{SO}_{3}{ }^{-}\right), 2.55$ (backbone $-\mathrm{CH}<$ ), $2.03\left(-\mathrm{N}^{+}\left(\mathrm{CH}_{3}\right)_{2} \mathrm{CH}_{2} \mathrm{CH}_{2} \mathrm{CH}_{2}{ }^{-}\right.$and backbone $\left.-\mathrm{CH}_{2}-\right), \quad 1.85$ $\left(-\mathrm{N}^{+}\left(\mathrm{CH}_{3}\right)_{2} \mathrm{CH}_{2} \mathrm{CH}_{2} \mathrm{CH}_{2}-\right)$.

RAFT polymerization of MA2-3 with an activated ester end group (procedure C). Monomer MA2-3 (1 g, $3.58 \mathrm{mmol}$, 250 equiv.), RAFT agent pentafluorophenyl 4-cyano-4-((phenylcarbonothioyl)thio)valerate (6.4 mg, $14.4 \mu \mathrm{mol}, 1$ equiv.), activated ester-functional initiator bis(pentafluorophenyl) 4,4'-(diazene-1,2-diyl)bis(4-cyanopentanoate) $(0.9 \mathrm{mg}, 1.44 \mu \mathrm{mol}$, 0.1 equiv.) and anhydrous propylene carbonate $(7 \mathrm{~mL})$ were combined and polymerized and purified as described above. After cooling to room temperature, to $4 \mathrm{~mL}$ of polymer solution was added 4-nitro-7-piperazin-1-yl-2,1,3-benzoxadiazole $(6.7 \mathrm{mg}, 26.9 \mu \mathrm{mol}, 3.4$-fold excess with regards to PFP esters) and the mixture was stirred for 1 day at RT, followed by addition of brine and extraction with ether and dialysis in ultrapure water at $35{ }^{\circ} \mathrm{C}$. The resulting polymer was dried yielding an orange solid that exhibited UCST behaviour in water, qualitatively confirming attachment of dye to the polymer.

Poly(pentafluorophenyl acrylate), pPFPA. Monomer PFPA (5.0 g, 21.0 mmol, 250 equiv.), RAFT agent BPTC (20.4 mg, $0.084 \mathrm{mmol}, 1$ equiv.), initiator $\mathrm{AIBN}(1.4 \mathrm{mg}, 8.4 \mu \mathrm{mol}, 0.1$ equiv.) and acetonitrile $(7 \mathrm{~mL})$ were combined in a flask equipped with a stir bar. The mixture was sealed with a rubber septum and purged with nitrogen for 30 minutes before being placed into a preheated oil bath at $70{ }^{\circ} \mathrm{C}$ for $10 \mathrm{~h}$. Polymerization was stopped by quenching the reaction with liquid nitrogen. A sample $(100 \mu \mathrm{L})$ was withdrawn, diluted with $\mathrm{CDCl}_{3}$ $(550 \mu \mathrm{L})$ and analysed by ${ }^{19} \mathrm{~F}$ NMR spectroscopy which indicated a monomer conversion of $71 \%$ by comparison of the signal at $-156.9 \mathrm{ppm}$ (bs, polymer para-F) with the signal at $-158.0 \mathrm{ppm}$ ( $\mathrm{t}$, monomer para-F). The polymer was isolated as a slightly yellow powder $(2.73 \mathrm{~g}, 55 \%)$ by two precipitations in methanol followed by drying in vacuum. $M_{\mathrm{n}}^{\text {theor. }}$ (from conversion) $=42.6 \mathrm{~kg} \mathrm{~mol}^{-1}, \mathrm{DP}_{\text {theor. }}=178, M_{\mathrm{n}}^{\mathrm{SEC}}=68.6 \mathrm{~kg} \mathrm{~mol}^{-1}$ (PS standard), $\bigoplus_{\mathrm{M}}=M_{\mathrm{w}, \mathrm{SEC}} / M_{\mathrm{n}, \mathrm{SEC}}=1.23 ;{ }^{19} \mathrm{~F} \mathrm{NMR}(282 \mathrm{MHz}$, $\mathrm{CDCl}_{3}$ ), $\delta / \mathrm{ppm}=-153.2$ (bm, $2 \mathrm{~F}$, ortho), -156.8 (bs, $1 \mathrm{~F}$, para), -162.2 (bs, 2 F, meta). FT-IR: $\nu / \mathrm{cm}^{-1}=1780$ (carbonyl $\mathrm{C}=\mathrm{O}$ stretch), 1520 (aryl $\mathrm{C}=\mathrm{C}$ bend).

3-((3-Aminopropyl)dimethylammonio)propane-1-sulfonate, (amine3-3) was prepared in 3 steps as previously reported: ${ }^{43}$ (i) protection of the primary amino group of $\mathrm{N}, \mathrm{N}$-dimethylaminopropylamine with di-tert-butyl dicarbonate $\left(\mathrm{BOC}_{2} \mathrm{O}\right)$ yielding tert-butyl (3-(dimethylamino)propyl)carbamate, which was purified by column chromatography in chloroformmethanol $6: 1$; (ii) quaternization of the tertiary amino group with 1,3-propanesultone and (iii) cleavage of the BOC protecting group with acid followed by treatment with base to give the free base product.

4-((3-Aminopropyl)dimethylammonio)butane-1-sulfonate, (amine3-4) was prepared in analogy to amine3-3 by substituting 1,3-propanesultone with 1,4-butanesultone in step (ii) under modified reaction conditions: tert-Butyl (3-(dimethylamino)propyl)carbamate (2.5 g, $12 \mathrm{mmol}, 1$ equiv.) was dissolved in anhydrous THF (15 mL), the flask was flushed with 
nitrogen, and 1,4-butanesultone (1.8 mL, $17 \mathrm{mmol}, 1.4$ eq.) was added. The mixture was stirred for 4 days at $40{ }^{\circ} \mathrm{C}$. The precipitated white solid was filtered off, washed with THF $(100 \mathrm{~mL})$, and dried in vacuum at $40{ }^{\circ} \mathrm{C}$. Yield: $1.95 \mathrm{~g}$, (48\%). ${ }^{1} \mathrm{H}$ NMR $\left(300 \mathrm{MHz}, \mathrm{D}_{2} \mathrm{O}\right), \delta / \mathrm{ppm}=3.35(\mathrm{~m}, 4 \mathrm{H}$, $\left.-\mathrm{CH}_{2} \mathrm{~N}^{+}\left(\mathrm{CH}_{3}\right)_{2} \mathrm{CH}_{2}-\right), 3.20\left(\mathrm{t}, 2 \mathrm{H},-\mathrm{CH}_{2} \mathrm{CH}_{2} \mathrm{NHBOC}\right), 3.09$ $\left(\mathrm{s}, 6 \mathrm{H},-\left(\mathrm{CH}_{3}\right)_{2} \mathrm{~N}^{+}-\right), 2.99\left(\mathrm{t}, 2 \mathrm{H},{ }^{-} \mathrm{O}_{3} \mathrm{SCH}_{2}-\right), 2.02-1.46$ $\left(\mathrm{m}, 6 \mathrm{H},{ }^{-} \mathrm{O}_{3} \mathrm{SCH}_{2} \mathrm{CH}_{2} \mathrm{CH}_{2}-,-\mathrm{N}^{+}\left(\mathrm{CH}_{3}\right)_{2} \mathrm{CH}_{2} \mathrm{CH}_{2}-\right), 1.46(\mathrm{~s}, 9 \mathrm{H}$, $\left.-\mathrm{C}\left(\mathrm{CH}_{3}\right)_{3}\right) \cdot{ }^{13} \mathrm{C}$ NMR $\left(300 \mathrm{MHz}, \mathrm{D}_{2} \mathrm{O}\right), \delta / \mathrm{ppm}=158.14(>\mathrm{C}=\mathrm{O})$, $81.21\left(-\mathrm{C}\left(\mathrm{CH}_{3}\right)_{3}\right), \quad 63.35,61.67\left(-\mathrm{CH}_{2} \mathrm{~N}^{+}\left(\mathrm{CH}_{3}\right)_{2} \mathrm{CH}_{2}-\right), \quad 50.74$ $\left(-\mathrm{N}^{+}\left(\mathrm{CH}_{3}\right)_{2}-\right), \quad 49.95 \quad\left({ }^{-} \mathrm{O}_{3} \mathrm{SCH}_{2}-\right), 36.82 \quad\left(-\mathrm{CH}_{2} \mathrm{CH}_{2} \mathrm{NHBOC}\right)$, $27.63\left(-\mathrm{C}\left(\mathrm{CH}_{3}\right)_{3}\right), \quad 22.68\left(-\mathrm{N}^{+}\left(\mathrm{CH}_{3}\right)_{2} \mathrm{CH}_{2} \mathrm{CH}_{2}-\right), \quad 21.06$ $\left({ }^{-} \mathrm{O}_{3} \mathrm{SCH}_{2} \mathrm{CH}_{2} \mathrm{CH}_{2}-\right), 20.82\left({ }^{-} \mathrm{O}_{3} \mathrm{SCH}_{2} \mathrm{CH}_{2}-\right)$. After treatment with aq. $\mathrm{HCl}$ and anion exchange beads, $0.99 \mathrm{~g}$ (74\%) free base amine3-4 was obtained. ${ }^{1} \mathrm{H}$ NMR $\left(300 \mathrm{MHz}, \mathrm{D}_{2} \mathrm{O}\right), \delta / \mathrm{ppm}=$ 3.39-3.32 (m, $\left.4 \mathrm{H},-\mathrm{CH}_{2} \mathrm{~N}^{+}\left(\mathrm{CH}_{3}\right)_{2} \mathrm{CH}_{2}-\right), 3.09(\mathrm{~s}, 6 \mathrm{H}$, $\left.-\mathrm{N}^{+}\left(\mathrm{CH}_{3}\right)_{2}{ }^{-}\right), 2.99\left(\mathrm{t}, 2 \mathrm{H},{ }^{-} \mathrm{O}_{3} \mathrm{SCH}_{2}-\right), 2.72\left(\mathrm{t}, 2 \mathrm{H},-\mathrm{CH}_{2} \mathrm{NH}_{2}\right)$, 2.01-1.77 (m, $\left.6 \mathrm{H},-\mathrm{CH}_{2} \mathrm{CH}_{2} \mathrm{CH}_{2} \mathrm{~N}^{+}\left(\mathrm{CH}_{3}\right)_{2} \mathrm{CH}_{2} \mathrm{CH}_{2} \mathrm{CH}_{2} \mathrm{NH}_{2}\right)$. ${ }^{13} \mathrm{C} \quad \mathrm{NMR}\left(300 \mathrm{MHz}, \mathrm{D}_{2} \mathrm{O}\right), \delta / \mathrm{ppm}=63.47, \quad 62.20$ $\left(-\mathrm{CH}_{2} \mathrm{~N}^{+}\left(\mathrm{CH}_{3}\right)_{2} \mathrm{CH}_{2}-\right), 50.63\left(-\mathrm{N}^{+}\left(\mathrm{CH}_{3}\right)_{2}{ }^{-}\right), 49.93\left({ }^{-} \mathrm{O}_{3} \mathrm{SCH}_{2}-\right)$, $\left.37.61 \quad\left(-\mathrm{CH}_{2} \mathrm{CH}_{2} \mathrm{NH}_{2}\right), \quad 24.96-\mathrm{CH}_{2} \mathrm{CH}_{2} \mathrm{NH}_{2}\right), \quad 21.07$ $\left({ }^{-} \mathrm{O}_{3} \mathrm{SCH}_{2} \mathrm{CH}_{2} \mathrm{CH}_{2}-\right), 20.80\left({ }^{-} \mathrm{O}_{3} \mathrm{SCH}_{2} \mathrm{CH}_{2}{ }^{-}\right)$.

General procedure for postpolymerization modification of pPFPA with zwitterionic amines. pPFPA (44.7 mg, $0.188 \mathrm{mmol}$ of repeat units, 1 equiv.) was dissolved in anhydrous propylene carbonate $(1.5 \mathrm{~mL})$ at $60{ }^{\circ} \mathrm{C}$ and hydroxyethyl acrylate $(5 \mu \mathrm{L})$ was added to scavenge thiols released from the RAFT end groups through aminolysis. ${ }^{50}$ In parallel, 3-((3-aminopropyl)dimethylammonio)propane-1-sulfonate, (amine3-3, $63.2 \mathrm{mg}$, $0.282 \mathrm{mmol}, 1.5$ equiv.) or 4 -((3-aminopropyl)dimethylammonio)butane-1-sulfonate, (amine3-4, $67.1 \mathrm{mg}, 0.282 \mathrm{mmol}$, 1.5 equiv.) was dissolved in propylene carbonate $(1.5 \mathrm{~mL})$ with heating. After dissolving, the amine solution was quickly added into the polymer solution and the mixture stirred at $40{ }^{\circ} \mathrm{C}$ overnight. A sample $(100 \mu \mathrm{L})$ was withdrawn, diluted with DMSO $(550 \mu \mathrm{L})$ and analysed by ${ }^{19} \mathrm{~F}$ NMR spectroscopy indicating complete conversion of PFP esters showing only signals of free pentafluorophenol at $\delta / \mathrm{ppm}=-167.4(2 \mathrm{~F}$, ortho), -170.2 (2 F, meta), -181.7 (1 F, para). The solution was transferred into a dialysis bag (molecular weight cut-off $3500 \mathrm{~g} \mathrm{~mol}^{-1}$ ) and dialyzed against ultrapure water for 3 days at RT (p(Am3-3)) or $60{ }^{\circ} \mathrm{C}(\mathbf{p}(\mathbf{A m} 3-4))$, followed by freeze-drying.

Poly[3-((3-acrylamidopropyl)dimethylammonio)propane-1sulfonate], p(Am3-3): ${ }^{1} \mathrm{H}$ NMR ( $\left.{ }_{2} \mathrm{O}, 500 \mathrm{mM} \mathrm{NaCl}, 300 \mathrm{MHz}\right)$ $\delta / \mathrm{ppm}=3.55\left(\mathrm{bs},-\mathrm{N}^{+}\left(\mathrm{CH}_{3}\right)_{2} \mathrm{CH}_{2} \mathrm{CH}_{2}-\right), 3.43$ (bs, $-\mathrm{NHCH}_{2} \mathrm{CH}_{2} \mathrm{CH}_{2}-$ ), 3.28 (bs, $-\mathrm{NHCH}_{2} \mathrm{CH}_{2} \mathrm{CH}_{2}-$ ), 3.18 (bs, $-\mathrm{N}^{+}\left(\mathrm{CH}_{3}\right)_{2}-$ ), 3.03 (bt, ${ }_{-} \mathrm{CH}_{2} \mathrm{SO}_{3}{ }^{-}$), $2.26\left(\mathrm{bs},-\mathrm{N}^{+}\left(\mathrm{CH}_{3}\right)_{2} \mathrm{CH}_{2} \mathrm{CH}_{2}{ }^{-}\right.$), 2.11, 2.07 (backbone $-\mathrm{CH}<$ ) and $\left(-\mathrm{NHCH}_{2} \mathrm{CH}_{2} \mathrm{CH}_{2}-\right)$, 1.76, 1.66 (backbone $\left.-\mathrm{CH}_{2}-\right)$.

Poly[4-((3-acrylamidopropyl)dimethylammonio)butane1-sulfonate], p(Am3-4): ${ }^{1} \mathrm{H}$ NMR $\left(\mathrm{D}_{2} \mathrm{O}, 500 \mathrm{mM} \mathrm{NaCl}\right.$, $300 \mathrm{MHz}), \delta / \mathrm{ppm}=3.40\left(\mathrm{bs},-\mathrm{N}^{+}\left(\mathrm{CH}_{3}\right)_{2} \mathrm{CH}_{2} \mathrm{CH}_{2} \mathrm{CH}_{2}-\right.$, $-\mathrm{NHCH}_{2} \mathrm{CH}_{2} \mathrm{CH}_{2}^{-}$), 3.28 (bs, $-\mathrm{NHCH}_{2} \mathrm{CH}_{2} \mathrm{CH}_{2}-$ ), 3.15 (bs, $\left.-\mathrm{N}^{+}\left(\mathrm{CH}_{3}\right)_{2} \mathrm{CH}_{2} \mathrm{CH}_{2} \mathrm{CH}_{2}{ }^{-}\right), 3.01$ (bt, ${ }_{-} \mathrm{CH}_{2} \mathrm{SO}_{3}{ }^{-}$), 2.02 (bs, $\left.-\mathrm{N}^{+}\left(\mathrm{CH}_{3}\right)_{2} \mathrm{CH}_{2} \mathrm{CH}_{2} \mathrm{CH}_{2}-,-\mathrm{N}^{+}\left(\mathrm{CH}_{3}\right)_{2} \mathrm{CH}_{2} \mathrm{CH}_{2} \mathrm{CH}_{2}-\right)$, overlapping 2.11 (backbone $-\mathrm{CH}<$ ), 1.86 (bm, $-\mathrm{NHCH}_{2} \mathrm{CH}_{2} \mathrm{CH}_{2}-$ ), 1.64 (backbone $-\mathrm{CH}_{2}^{-}$).
General procedure for postpolymerization modification of pPFPA with zwitterionic amine and benzylamine. Poly(pentafluorophenyl acrylate), pPFPA $(44.7 \mathrm{mg}, 0.188 \mathrm{mmol}$ of repeat units, 1 equiv.) was dissolved in anhydrous propylene carbonate $(1.5 \mathrm{~mL})$ at $60{ }^{\circ} \mathrm{C}$, and hydroxyethyl acrylate $(5 \mu \mathrm{L})$ was added. In parallel, 4-((3-aminopropyl)dimethylammonio) butane-1-sulfonate, (amine3-4) $(x \times 0.282 \mathrm{mmol})$ and benzylamine $(y \times 0.282 \mathrm{mmol}, x+y=1 ; 1.5$ equiv. of amines to PFP ester) were dissolved in propylene carbonate $(1.5 \mathrm{~mL})$ with heating. After dissolving, the amine solution was quickly added into the polymer solution and the mixture stirred at $40{ }^{\circ} \mathrm{C}$ overnight. Analysis of the reaction mixture was done as described above for the corresponding homopolymers. Products were isolated by dialysis first in $500 \mathrm{mM}$ aqueous $\mathrm{NaCl}$ solution for 2 days, then ultrapure water at $60{ }^{\circ} \mathrm{C}$ for 3 days. ${ }^{1} \mathrm{H}$ NMR ( $\mathrm{D}_{2} \mathrm{O}, 500 \mathrm{mM} \mathrm{NaCl}, 300 \mathrm{MHz}$, benzyl side groups), $\delta / \mathrm{ppm}=7.53,7.29\left(\mathrm{~m}, \quad-\mathrm{CH}_{2} \mathrm{C}_{6} \mathrm{H}_{5}\right), 4.45 \quad\left(-\mathrm{CH}_{2} \mathrm{C}_{6} \mathrm{H}_{5}\right)$. Molar compositions of the benzylacrylamide copolymers poly [(4-((3-acrylamidopropyl)dimethylammonio)butane-1-sulfonate $)_{x}$ co-benzylacrylamide $y], \mathbf{p}\left[(\mathbf{A m} \mathbf{3}-\mathbf{4})_{\boldsymbol{x}} \boldsymbol{-} \boldsymbol{c o}-\mathbf{B z}_{\boldsymbol{y}}\right]$ were determined by comparison of the aromatic peaks $(5 \mathrm{H})$ to the sum of the zwitterionic group signals from 3.76-2.74 (14 H).

\section{Results and discussion}

In this work, zwitterionic monomers and their corresponding (co)polymers are abbreviated based on their polymerizable group, i.e. acrylate (A), methacrylate (MA), acrylamide (Am), and methacrylamide (MAm), followed by the spacer length between polymerizable unit and ammonium group (2 or 3 ) and the ion bridge spacer length (3 for SPB, 4 for SBB), see Scheme 1.

\section{Method 1: quaternization of tertiary amine functional polymers with sultones}

In order to compare the UCST behaviour of SBB with SPB polymers with identical degrees of polymerization, postmodification of tertiary amine functional species with 1,3propanesultone (PS) and 1,4-butanesultone (BS) was considered first. Arguably the most popular tertiary amine-functional homopolymer is pDMAEMA. Notably, its acrylate sister polymer poly[2-(dimethylamino)ethyl acrylate] suffers from hydrolytic instability ${ }^{51}$ and is far less commonly employed; this method toward SPB and SBB polymers thus primarily gives access to the methacrylic species p(MA2-3) and p(MA2-4), see Scheme 2.

RAFT-made pDMAEMA (DP $=201, D_{\mathrm{M}}=M_{\mathrm{w}} / M_{\mathrm{n}}=1.14$ ) was reacted under homogeneous conditions in 2,2,2-trifluoroethanol (TFE) as solvent with 2 equiv. (with respect to amino groups) of PS for 3 days at $40{ }^{\circ} \mathrm{C}$ and with 2 equiv. of BS for 4 days under reflux. Excess reagent was removed by adding brine and washing with diethyl ether and product polymers were isolated by dialysis against ultrapure water and analysed by ${ }^{1} \mathrm{H}$ NMR spectroscopy, aqueous size exclusion chromatography (SEC), and turbidity measurements. While the NMR spectrum 


\begin{tabular}{|c|c|c|c|c|c|}
\hline & & & & & $\begin{array}{ll}\mathrm{R}_{1}=\mathrm{H}, \mathrm{CH}_{3} & \mathrm{p}=2,3 \\
\mathrm{Y}=\mathrm{O}, \mathrm{NH} & \mathrm{q}=3,4\end{array}$ \\
\hline Code & $\mathbf{R}_{\mathbf{1}}$ & $\mathbf{Y}$ & $\mathbf{p}$ & $\mathbf{q}$ & Full Name \\
\hline p(MA2-3) & $\mathrm{CH}_{3}$ & $\mathrm{O}$ & 2 & 3 & poly[3-((2-(methacryloyloxy)ethyl)dimethylammonio)propane-1-sulfonate] \\
\hline p(MA2-4) & $\mathrm{CH}_{3}$ & $\mathrm{O}$ & 2 & 4 & poly[4-((2-(methacryloyloxy)ethyl)dimethylammonio)butane-1-sulfonate $]$ \\
\hline p(A2-4) & $\mathrm{H}$ & $\mathrm{O}$ & 2 & 4 & poly[4-((2-(acryloyloxy)ethyl)dimethylammonio)butane-1-sulfonate] \\
\hline p(Am3-3) & $\mathrm{H}$ & $\mathrm{NH}$ & 3 & 3 & poly[3-((3-acrylamidopropyl)dimethylammonio)propane-1-sulfonate] \\
\hline p(Am3-4) & $\mathrm{H}$ & $\mathrm{NH}$ & 3 & 4 & poly[4-((3-acrylamidopropyl)dimethylammonio)butane-1-sulfonate] \\
\hline p(MAm3-4) & $\mathrm{CH}_{3}$ & NH & 3 & 4 & poly[4-((3-methacrylamidopropyl)dimethylammonio)butane-1-sulfonate] \\
\hline
\end{tabular}

Scheme 1 General structure of common sulfobetaine polymers and details and full names of polymers prepared in this study through different synthetic methods.

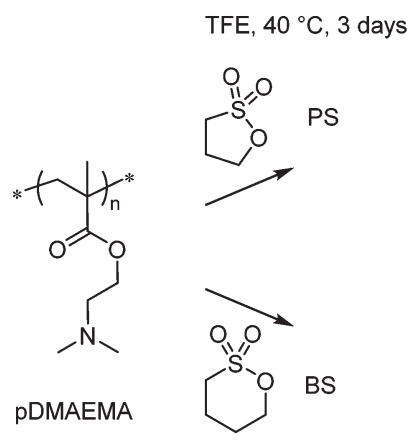

TFE, reflux, 4 days
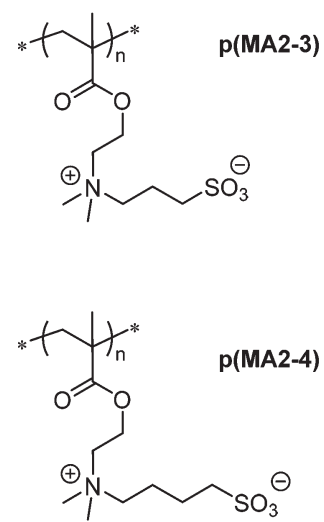

Scheme 2 Method 1: synthesis of polysulfobetaines through postmodification of poly[2-(dimethylamino)ethyl methacrylate] (pDMAEMA) with PS and BS in 2,2,2-trifluoroethanol (TFE).

of the product from pDMAEMA and PS, Fig. 1A, conformed to the expected homo-p(MA2-3), suggesting quantitative conversion, the modification of PDMAEMA with the less reactive BS was found to be only $95 \%$ complete, as judged from a residual broad singlet at $2.64 \mathrm{ppm}$ in the ${ }^{1} \mathrm{H}$ NMR spectrum (Fig. 1B) which was assigned to the $\left(\mathrm{CH}_{3}\right)_{2} \mathrm{~N}$ - resonance of unreacted DMAEMA segments. Aqueous SEC revealed narrow molecular weight distributions of $\mathbf{p}(\mathbf{M A 2}-3)$ and $\mathbf{p}\left[(\mathbf{M A 2}-\mathbf{4})_{\mathbf{0 . 9 5}}-\mathbf{c o}-\right.$ DMAEMA $_{\mathbf{0 . 0 5}}$ ] predictably similar to that of the pDMAEMA parent, see Fig. 2A and Table 1. The zwitterionic (co)polymers eluted at a similar time, suggesting similar hydrodynamic sizes in the $0.2 \mathrm{M} \mathrm{NaCl}$ eluent. The p(MA2-3) sample was found to be soluble in ultrapure water over the entire observed temperature range from $0-90{ }^{\circ} \mathrm{C}$ at a concentration of $10 \mathrm{~g} \mathrm{~L}^{-1}$, not exhibiting the anticipated UCST behaviour. Presumably, in spite of the favourable ${ }^{1} \mathrm{H}$ NMR measurement (Fig. 1A), the modification may not have been quantitative or the pDMAEMA precursor may have suffered hydrolysis/alcoholysis during the extended reaction time. Incomplete betainization of PDMAEMA has been shown to lead to enhanced water solubility (i.e. decreased UCST transitions) due to the hydrophilicity associated with the residual DMAEMA repeat units. ${ }^{52}$ The butane-spaced sister copolymer $\mathbf{p}\left[(\mathbf{M A 2}-\mathbf{4})_{\mathbf{0 . 9 5}}\right.$-co-DMAEMA $\left.\mathbf{0 . 0 5}_{\mathbf{0}}\right]$, on the other hand and regardless of its incomplete degree of betainization, exhibited a high cloud point of $77.9^{\circ} \mathrm{C}$.

Reduced solubility of SBB species compared to analogous SPBs has been documented for small molecule zwitterionic surfactants and has been speculated to be due to higher hydrophobicity imparted by the additional methylene group in the spacer. ${ }^{26,53,54}$ Bredas et al. ${ }^{27}$ found in a theoretical assessment of the aggregation of zwitterionic SPB and SBB end groups in non-polar solvents that SBB head groups (which, same as SPB groups, are usually curled as opposed to in an all-trans conformation $)^{28,29}$ can more easily adopt an extended conformation when aggregating which makes their dimerization (and aggregation) more favourable than that of SPB groups. To the best of our knowledge, reduced solubility of SBB-functional polymers compared to their SPB counterparts has only very recently been described. ${ }^{22}$ Given the significantly higher cloud point of the (albeit incompletely modified) MA2-4 copolymer and the resulting potential for UCST tuning (as elaborated above, a reduction of UCST values is easiest to achieve), other routes to SBB (co)polymers were examined. 
<smiles>CC(C)(C)C(Cl)(CCCS(=O)(=O)[O-])C(=O)OCC[N+](C)(Cl)Cl</smiles>

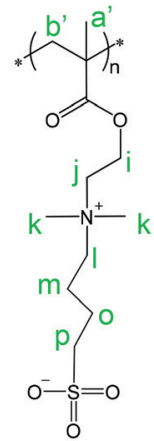

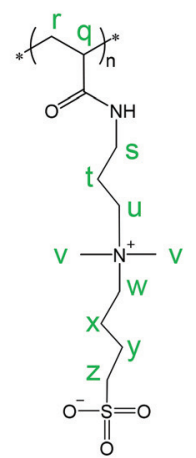
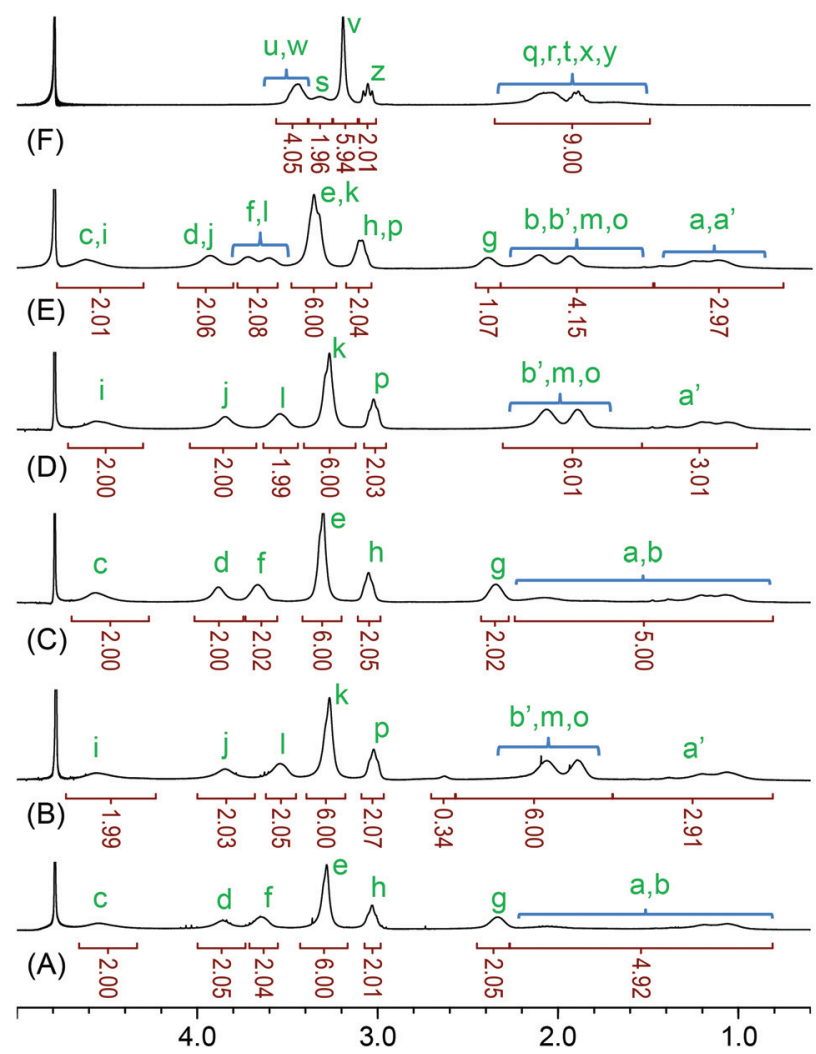

Fig. $1{ }^{1} \mathrm{H}$ NMR spectra measured in $\mathrm{D}_{2} \mathrm{O}$ containing $500 \mathrm{mM} \mathrm{NaCl}$ of (A) $p(M A 2-3)$ prepared from pDMAEMA (method 1); (B) $p(M A 2-4)$ prepared from pDMAEMA (method 1); (C) $p$ (MA2-3) prepared by RAFT polymerization (method 2); (D) p(MA2-4) prepared by RAFT polymeri-

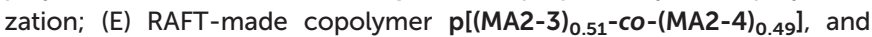
(F) $\mathrm{p}(\mathrm{Am} 3-4)$ obtained from postpolymerization modification of pPFPA (method 3).

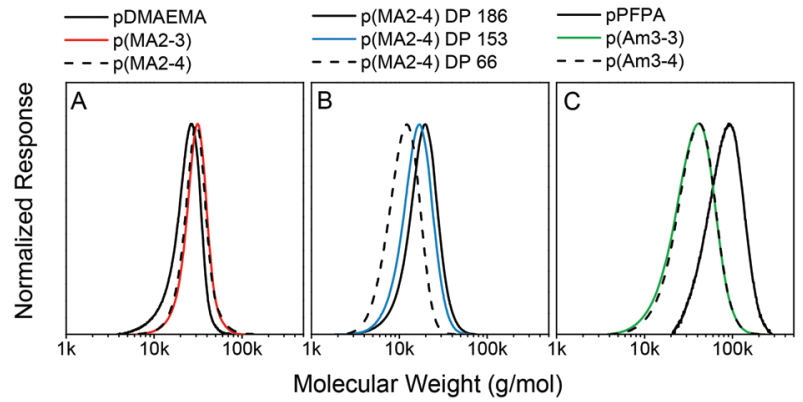

Fig. 2 SEC curves of (A) pDMAEMA precursor (eluent DMAc, polystyrene standards) and its daughter homopolymers $p(M A 2-3)$ and p(MA2-4) (eluent $0.2 \mathrm{M}$ aqueous $\mathrm{NaCl}, \mathrm{PEG}$ standards) (method 1); (B) RAFT-made $\mathrm{p}(\mathrm{MA2}-4)$ of different degrees of polymerization (eluent 0.2 $\mathrm{M}$ aqueous $\mathrm{NaCl}$, PEG standards) (method 2); and (C) pPFPA precursor (eluent DMAc, polystyrene standards) and its daughter homopolymers $\mathrm{p}(\mathrm{Am} 3-3)$ and $\mathrm{p}(\mathrm{Am} 3-4)$ (eluent $0.2 \mathrm{M}$ aqueous $\mathrm{NaCl}, \mathrm{PEG}$ standards) (method 3).

\section{Method 2: RAFT (co)polymerization of zwitterionic monomers}

Polymerization of zwitterionic monomers yields zwitterionic polymers in one step. In addition to monomer MA2-3 used for the preparation of reference polymers, the SBB species MA2-4, A2-4 and MAm3-4 were synthesized in analogy to literature procedures $^{18,49,55}$ from the respective dimethylamino-functional monomers in acetonitrile with BS in moderate to high yields, Scheme 3.

RAFT polymerization ${ }^{56}$ in homogeneous TFE solution using dithioester and trithiocarbonate chain transfer agents (CTAs) was employed to access (co)polymers with predetermined molecular weights with narrow molecular weight distributions, see Scheme 4. Table 2 summarizes conversions, degrees of polymerization (DP, calculated from feed and conversion), aqueous SEC results and cloud points for a series of MA2-3 homopolymers (entries 1-3), a series of MA2-4 homopolymers (entries 5-7), a p(A2-4) species (entry 8), and a series of statistical copolymers $\mathbf{p}\left[(\mathbf{M A 2}-3)_{\boldsymbol{x}}\right.$-co-(MA2-4) $y$ ] with indices $x$ and $y$ denoting the respective measured molar percentages. Additionally, a sample of p(MA2-3) (entry 4 in Table 2) was prepared using a pentafluorophenyl (PFP)-functional CTA and PFP-functional initiator in propylene carbonate, structures are shown in Scheme 5.

Overall, the RAFT synthesis of zwitterionic SPB and SBB (co)polymers from MA2-3, MA2-4, and A2-4 proceeded

Table 1 Homopolymers derived from pDMAEMA ( $\left.D P^{a}=201, \Xi_{M}=M_{w} / M_{n}=1.14\right)$ by postpolymerization modification with sultones

\begin{tabular}{|c|c|c|c|c|c|c|c|}
\hline Product & Reagent & $\begin{array}{l}\text { Conversion }{ }^{b} \\
(\%)\end{array}$ & DP & $\begin{array}{l}M_{\mathrm{n}}^{\text {theor } c} \\
\left(\mathrm{~kg} \mathrm{~mol}{ }^{-1}\right)\end{array}$ & $\begin{array}{l}M_{\mathrm{n}}^{\mathrm{SEC} d} \\
\left(\mathrm{~kg} \mathrm{~mol} \mathrm{~mol}^{-1}\right)\end{array}$ & $D_{M}^{d}$ & $\begin{array}{l}\text { Cloud } \\
\text { point }^{e}\left({ }^{\circ} \mathrm{C}\right)\end{array}$ \\
\hline p(MA2-3) & 1,3-Propanesultone & 100 & 201 & 56.4 & 28 & 1.10 & $\mathrm{~S}^{f}$ \\
\hline
\end{tabular}

${ }^{a}$ Degree of polymerization calculated from conversion estimated by ${ }^{1} \mathrm{H}$ NMR measurement of polymerization mixture. ${ }^{b} \mathrm{Calculated}$ from ${ }^{1} \mathrm{H}$ NMR analysis. ${ }^{c}$ Molar mass calculated from DP. ${ }^{d}$ Poly(ethylene glycol) equivalent molecular weight and dispersity determined by aqueous SEC. ${ }^{e}$ UCST cloud point measured in ultrapure water at a concentration of $10 \mathrm{~g} \mathrm{~L}^{-1} .{ }^{f}$ Soluble between 0 and $90{ }^{\circ} \mathrm{C}$ at a concentration of $10 \mathrm{~g} \mathrm{\textrm {L } ^ { - 1 }}$. 


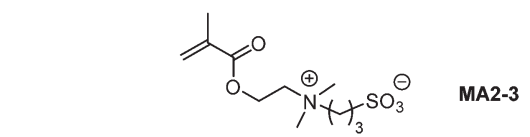<smiles>C=C(C)C(=O)OCCN(C)C</smiles><smiles>CC(C)(C)C1CCCS(=O)(=O)OC1</smiles><smiles>C=C(C)C(=O)OCC[N+](C)(C)C(C)(C)[Na]</smiles><smiles>C=CC(=O)OCCN(C)C</smiles><smiles>CC(C)(C)CC12CCCC(CCS(=O)(=O)O1)C2</smiles><smiles>C=CC(=O)OCCN(C)C</smiles>

A2-4<smiles>C=C(C)C(=O)NCCCN(C)C</smiles><smiles>CN(C)CC1CCCS(=O)(=O)O1</smiles><smiles>C=C(C)C(=O)NCCC[N+](C)(C)C(C)(C)C</smiles>

MAm3-4

Scheme 3 Overview of zwitterionic monomers including the commercially available species MA2-3 and sulfobutylbetaine (SBB) monomers prepared in this study.

smoothly in TFE with conversions generally ranging between $75-92 \%$ after polymerization at $70{ }^{\circ} \mathrm{C}$ overnight. (Co)polymer products had low measured dispersities of $\bigoplus_{M} \leq 1.23$, with the exception of one $\mathbf{p}$ (MA2-3) sample that proceeded to $99 \%$ monomer conversion and had a $D_{\mathrm{M}}$ of 1.37 . Exemplary molecular weight distribution curves obtained by aqueous SEC of the series of MA2-4 homopolymers are plotted in Fig. 2B showing increasing hydrodynamic sizes with increasing DPs. Since SEC yields only apparent molecular weights, the absolute molecular weight of an exemplary sample, p(MA2-4) with a calculated DP of 66 (Table 2, entry 5), was estimated by end group analysis. A solution of this polymer in 2,2,2-trifluoroethanol at a concentration of $1.0 \mathrm{~g} \mathrm{~L}^{-1}$ was found to have an absorbance maximum of $A=0.63$ at a wavelength of $308 \mathrm{~nm}$ which was attributed to a $\pi-\pi^{*}$ absorption band of the dithioester end group. ${ }^{57}$ Using the tabulated maximum molar absorptivity of a model RAFT agent, 4-cyano-4-(phenylcarbonothioylthio)pentanoic acid, of $\varepsilon=13200 \mathrm{~L} \mathrm{~mol}^{-1} \mathrm{~cm}^{-1}$, measured in methanol, ${ }^{57}$ as reference, an absolute molecular weight $M_{\mathrm{n}}=21.0 \mathrm{~kg}$ $\mathrm{mol}^{-1}$ was obtained, in reasonable agreement with the theoretical value of $19.6 \mathrm{~kg} \mathrm{~mol}^{-1}$. Notably, this calculation assumes (i) equal absorbivities of the dithioester group of the model RAFT agent in methanol and the dithioester end group of the zwitterionic polymer in 2,2,2-trifluoroethanol and (ii) every polymer chain has an intact dithioester end group. All ${ }^{1} \mathrm{H}$ NMR spectra conformed to the expected representative homo- and copolymer structures; Fig. 1 shows exemplary spectra of p(MA23) (spectrum C) and p(MA2-4) (spectrum D) samples and copolymer $\left.\mathbf{p}\left[(\mathbf{M A 2}-3)_{\mathbf{0 . 5 1}} \text {-co-(MA2-4) }\right)_{\mathbf{0 . 4 9}}\right]$. Note the striking similarity between spectra $\mathrm{C}$ and $\mathrm{A}$ and spectra $\mathrm{D}$ and $\mathrm{B}$, the latter with the exception of the above-mentioned residual DMAEMA

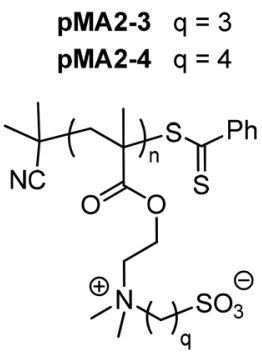<smiles>CCCSC(=S)SC(C)C(CCCc1ccccc1)C(=O)OCC[N+](C)(C)CCCCS(=O)(=O)[O-]</smiles>

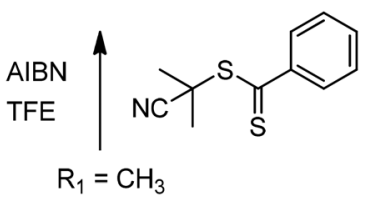<smiles>[R7]=CC1CCCCC1Cc1ccccc1</smiles>
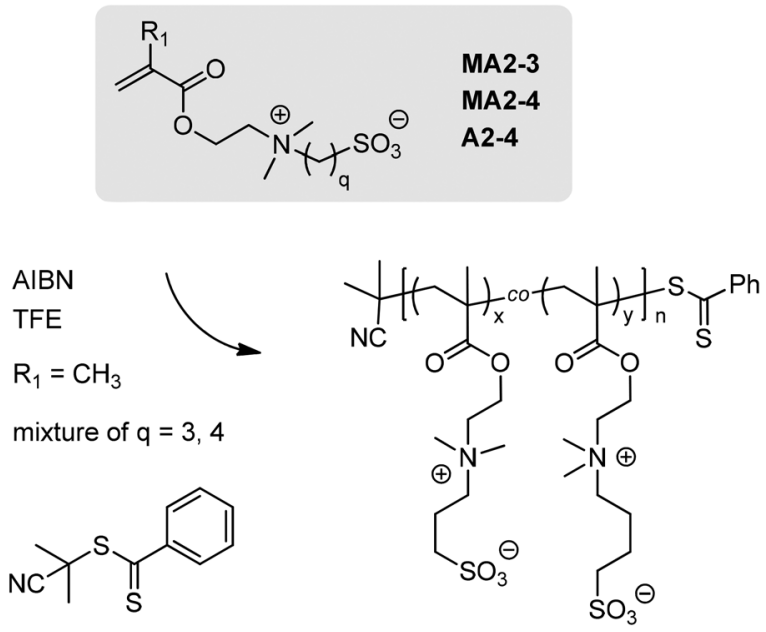

p[(MA2-3) $x-$-co-(MA2-4) $] \quad x+y=1$

Scheme 4 Method 2: Homo- and copolymerization of zwitterionic monomers by the RAFT process.

resonance. RAFT polymerization of the methacrylamide derivative MAm3-4, however, gave only very low conversions employing BPTC or CPBP as the mediating CTAs. Low conversion of the MAm3-3 analogue using a dithiobenzoate RAFT agent was previously observed by others; ${ }^{36}$ while Hildebrand et al., ${ }^{31}$ for example, described high conversions of MAm3-3 using a 4-cyano-4-[( phenylethylsulfanylthiocarbonyl)sulfanyl $]$ pentanoic acid derivative.

All RAFT-made (co)polymers showed sharp, fully reversible and reproducible UCST transitions with a hysteresis of $\sim 3{ }^{\circ} \mathrm{C}$, see Fig. S1 in the ESI. $\dagger$ The significant impact of the ion bridge length on the aqueous solution behaviour was apparent from a comparison of the $\mathbf{p}$ (MA2-4) series with the homologous p(MA2-3) reference series. A plot of their cloud points vs. their DP, shown in Fig. 3, supported the following observations: over the observed DP range, the UCST cloud point (CP) increases in a linear fashion with DP for both series. The CP increases more strongly with the DP for the SBB homopolymers, linear fits giving $\mathrm{CP}_{\mathrm{SBB}} /{ }^{\circ} \mathrm{C}=0.42 \times \mathrm{DP}-0.96$ for the 
Table 2 Homo- and copolymers prepared by RAFT radical polymerization of zwitterionic monomers

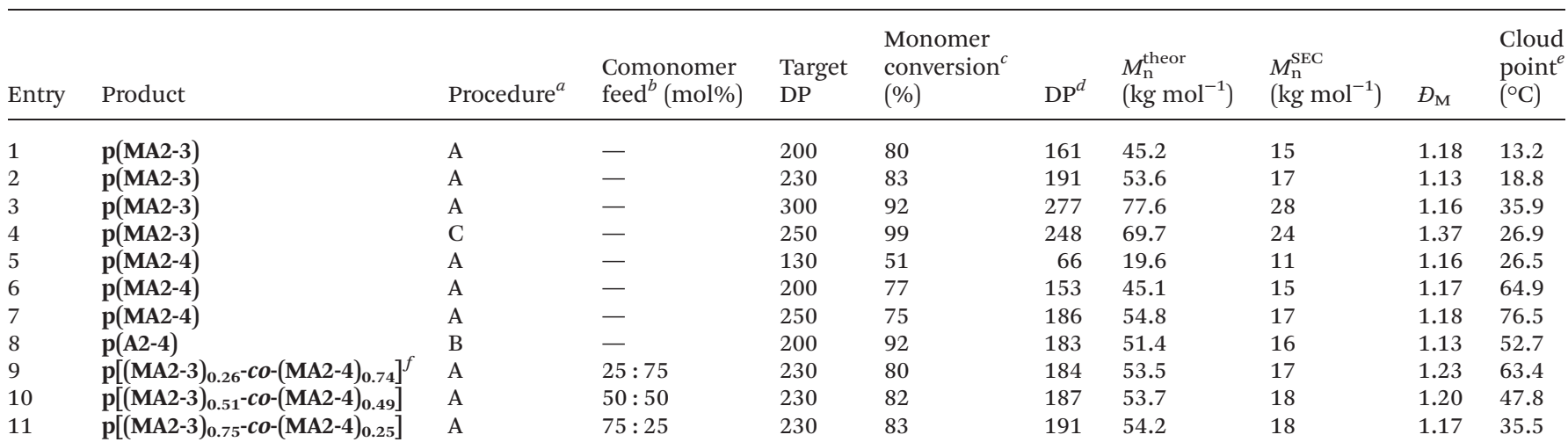

${ }^{a}$ Procedure A: CPDB in TFE; B: BPTC in TFE, C: PFP-functional CTA and initiator in propylene carbonate. ${ }^{b}$ Molar ratio of MA2-3-MA2-4 in feed. ${ }^{c}$ (Global) monomer conversion determined by ${ }^{1} \mathrm{H}$ NMR spectroscopy. ${ }^{d}$ Degree of polymerization estimated from conversion. ${ }^{e} \mathrm{UCST}$ cloud point measured in ultrapure water at a concentration of $10 \mathrm{~g} \mathrm{~L}^{-1} \cdot{ }^{f}$ Molar copolymer composition determined by ${ }^{1} \mathrm{H}$ NMR spectroscopy.

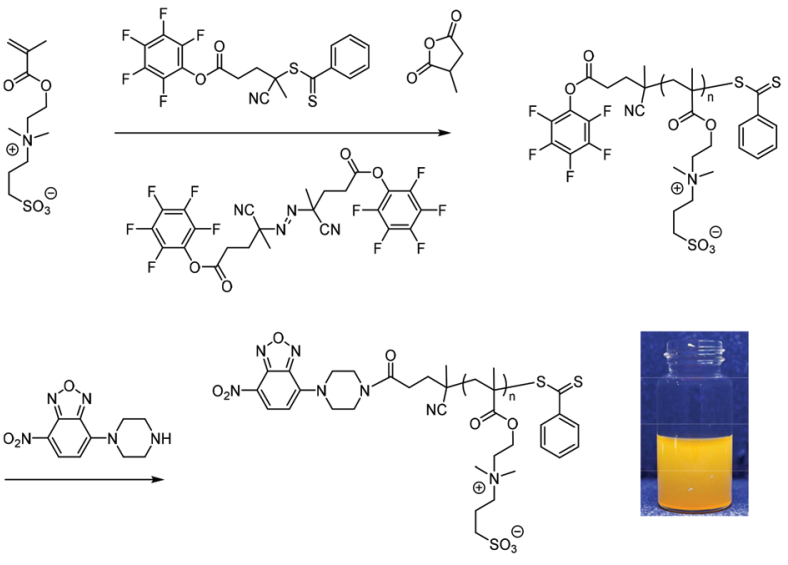

Scheme 5 RAFT polymerization of zwitterionic monomer MA2-3 using a PFP activated ester-functional CTA and a PFP-functional initiator followed by attachment of the dye 4-piperidyl-7-nitrobenzofurazan with inset photograph of the purified polymer solution under UV illumination.

$\mathbf{p}(\mathbf{M A 2}-4)$ series and $\mathrm{CP}_{\mathrm{SPB}} /{ }^{\circ} \mathrm{C}=0.20 \times \mathrm{DP}-18.5$ for the $\mathbf{p}(\mathbf{M A 2}-3)$ series. Thus, when comparing homopolymers of the same length, p(MA2-4) has significantly higher cloud points than p(MA2-3). The cloud point of the $\mathbf{p}$ (MA2-3) sample prepared with a different end group (entry 4 in Table 2 ) coincided with the trend of its sister polymers, indicating only small end group influence on the UCST transition at this DP. These observations suggest that, with regards to exploiting aqueous UCST behaviour, the investigated SBB species present a clear advantage over their SPB homologues. To reiterate, most manipulations of the aqueous solvent or the chemical structure of polysulfobetaines such as inclusion of hydrophilic (or certain hydrophobic) ${ }^{43}$ groups bring about a decrease of UCST cloud points necessitating scaffolds with fundamentally high UCST transitions in pure water. Judging from the plot in Fig. 3, aqueous UCST cloud points around body temperature

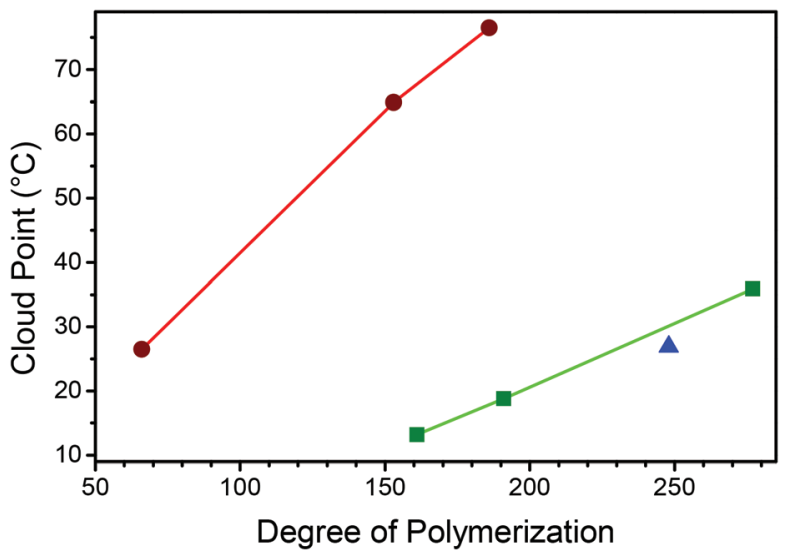

Fig. 3 UCST cloud point versus degree of polymerization for $p(M A 2-3)$ (green squares) and p(MA2-4) (red circles) prepared by RAFT in 2,2,2trifluoroethanol using $C P D B$ as chain transfer agent and of $p(M A 2-3)$ made by RAFT in propylene carbonate using the activated ester CTA (Scheme 5) (blue triangle). Lines are added to guide the eye.

can be realized with $\mathbf{p}$ (MA2-4) with an easily achievable DP of $\sim 100$, while, in agreement with literature, ${ }^{21,35}$ significantly higher DPs are necessary for p(MA2-3) to achieve similarly high cloud points.

The aqueous solution behaviour of the SBB acrylate species $\mathbf{p}(\mathbf{A 2}-4)(\mathrm{DP}=183)$ was also assessed and compared to $\mathbf{p}(\mathbf{M A 2}-\mathbf{4})$ $(\mathrm{DP}=186)$ and $\mathbf{p}(\mathbf{M A 2}-3)(\mathrm{DP}=191)$ with similar degrees of polymerization. A temperature-concentration phase diagram, showing the boundary between the two-phase (below the curve) and the one-phase region (above the curve), shown in Fig. 4, made evident the similar shape of the phase separation boundaries with flat regions above concentrations of $\sim 10 \mathrm{~g} \mathrm{~L}^{-1}$ for all three zwitterionic polymers. Of note, the acrylic polymer p(A2-4) exhibiting a cloud point of $52.7^{\circ} \mathrm{C}$ at a concentration of $10 \mathrm{~g} \mathrm{~L}^{-1}$ was more water soluble than the methacrylic analogue $\mathbf{p}$ (MA2-4) $\left(\mathrm{CP}=76.5^{\circ} \mathrm{C}\right.$ at the same concentration) but 


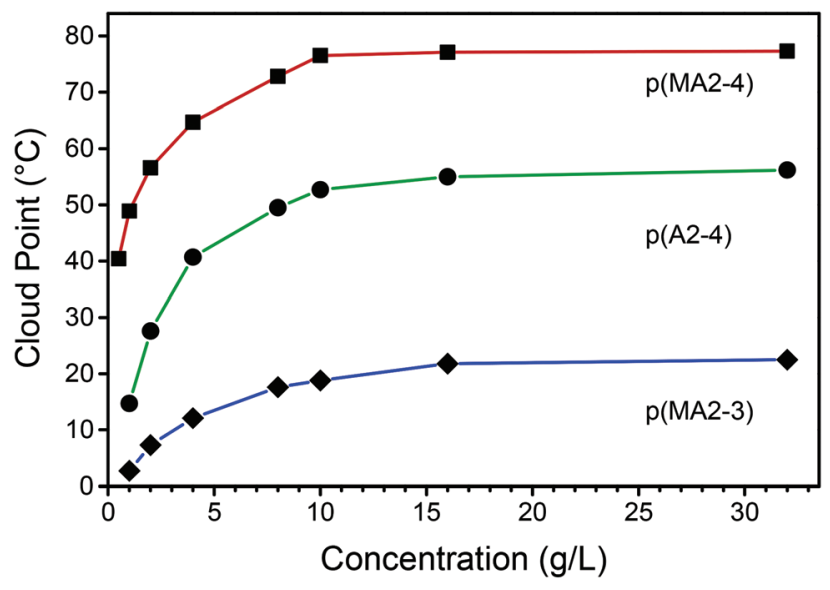

Fig. 4 Temperature-concentration phase diagrams of three RAFTmade homopolymers with comparable degrees of polymerization, $p(M A 2-4)(D P=186), p(A 2-4)(D P=183)$, and $p(M A 2-3)(D P=191)$ showing a largely flat region above concentration of $\sim 10 \mathrm{~g} \mathrm{~L}^{-1}$.

less soluble than the SPB derivative $\mathbf{p}(\mathbf{M A 2}-3)\left(\mathrm{CP}=18.8^{\circ} \mathrm{C}\right)$, indicating that the UCST is influenced much more sensitively by formal addition of a methylene segment to the ion bridge rather than to the backbone. This observation thus corroborated the notion of improved electrostatic interlocking efficiency of butyl-spaced zwitterionic groups due to higher flexibility of the of the butyl chains and their stronger tendency to adopt an extended conformation as compared to the propyl analogues. $^{27}$

Comparison of the RAFT-made homopolymer series with those obtained from post-modification of pDMAEMA suggested an influence of the synthetic method on the UCST behaviour. Judging from the linear fit of CP vs. DP for the RAFT-made p(MA2-3) series, Fig. 3, the pDMAEMA-made sample with a DP of 201 would be expected to have a CP of around $22{ }^{\circ} \mathrm{C}$, but was, as mentioned above, fully soluble. This comparison suggested that (for the chosen reaction conditions) the modification of PDMAEMA with sultones was not ideal with regards to obtaining (high) UCST transitions. In order to assess the scope and limitations of direct polymerization of zwitterionic monomers, two further experiments were conducted.

Firstly, the modification of polymer end groups is of great importance for the construction of defined polymeric architectures including diblock copolymers, surface-tethered brushes, or hybrid materials. RAFT polymerization was thus attempted with an activated ester CTA and activated ester initiator, as shown in Scheme 5. This approach produces polymers with one activated pentafluorophenol (PFP) end group providing access to simple modification with amines. ${ }^{46,58}$ The reactive CTA and initiator were, however, not soluble in water and were found to react, at elevated polymerization temperatures, with TFE. Therefore, propylene carbonate was used as a non-protic solvent in which monomer MA2-3 was moderately soluble, while, remarkably, MA2-4, was poorly soluble. Polymerization

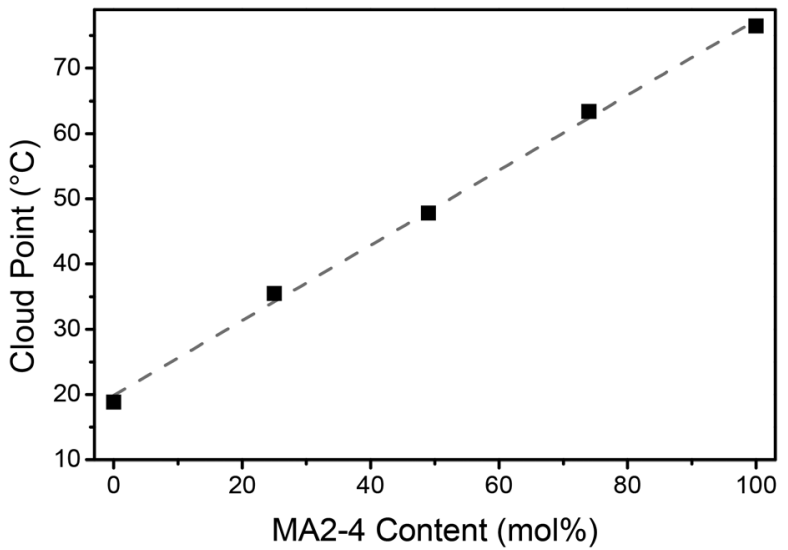

Fig. 5 Cloud point versus MA2-4 comonomer content for a series of statistical RAFT-made $\mathrm{p}\left[(\mathrm{MA2}-3)_{x}-\mathrm{co}-(\mathrm{MA2}-4)_{y}\right]$ copolymers and the two respective RAFT-made homopolymers $\mathrm{p}(\mathrm{MA2}-3)(y=0 \%)$ and (MA2-4) ( $y=100 \%$ ). All (co)polymers had comparable DPs ranging from 184-191. A linear fit (dashed curve) was added to guide the eye.

of MA2-3 thus yielded a homopolymer with a reactive end group which possessed UCST characteristics with a cloud point in good agreement with those of the series polymerized in TFE, see Fig. 3. Addition of an amine-functional dye to a propylene carbonate solution of the reactive end group-functional polymer followed by purification yielded an orangecoloured solid that dissolved in (warm) water with yellow colour, see Scheme 5. This experiment demonstrated successful RAFT polymerization of a zwitterionic SPB monomer with a reactive CTA in a non-protic solvent and provided qualitative evidence of successful end group modification with a hydrophobic dye. However, though advantageous with respect to higher UCST transitions, the low compatibility of the SBB MA2-4 with non-protic solvents poses limitations for the incorporation of water/TFE sensitive or insoluble species into MA24-based (co)polymers.

Secondly, a series of copolymers of MA2-3 and MA2-4 with comparable DPs (184-191) and SEC-measured apparent molar masses was prepared by RAFT radical polymerization in TFE. Copolymer compositions, determined by ${ }^{1} \mathrm{H}$ NMR spectroscopy (see Fig. 1E) and listed in Table 2, matched very well with the comonomer feed ratio, indicating the formation of copolymers with predetermined molar compositions. Determination of their aqueous solution behaviour revealed a linear increase of cloud points with MA2-4 comonomer content, Fig. 5, suggesting a convenient and simple methodology for tuning of UCST transitions at a constant chain length.

\section{Method 3: post-modification of activated ester precursors with zwitterionic amines}

Previously, we described the acyl substitution of poly(pentafluorophenyl acrylate) (pPFPA) with an amino-functional SPB, amine3-3 (shown in Scheme 6), as a versatile route toward series of SPB acrylamide (co)polymers with identical DPs including hydrophobically modified copolymers with tuneable 

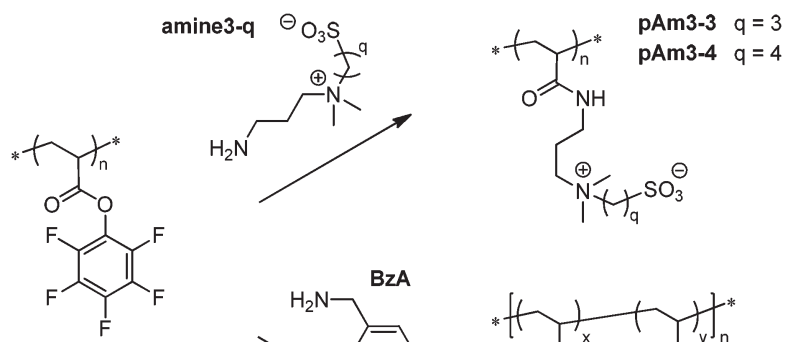

pPFPA
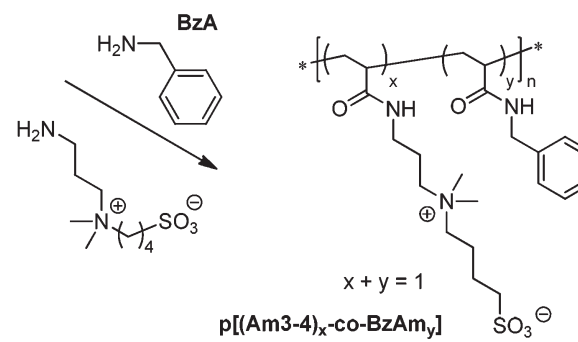

Scheme 6 Method 3: zwitterionic homo- and copolymers through postpolymerization modification of poly(pentafluorophenyl acrylate) ( $p P F P A)$ with zwitterionic amines and benzylamine.

UCSTs. $^{43}$ Key to this procedure was the solubility of all involved species-pPFPA precursor, zwitterionic amine, and (co)polymer product-in propylene carbonate (PC). In this approach the introduction of zwitterionic side groups does not rely on the reactivity of different sultones (as in the non-quantitative modification of pDMAEMA with BS giving ill-defined SBB species). Therefore, we next investigated the postpolymerization synthesis of SBB (co)polymers from polymeric activated ester precursors. For this purpose, a novel zwitterionic amine, 4-((3-aminopropyl)dimethylammonio)butane-1-sulfonate, amine3-4, was prepared in three steps starting from $\mathrm{N}, \mathrm{N}$-dimethylaminopropylamine by (i) BOC-protection of the primary amine, (ii) quaternization of the dimethylamino group with BS in THF, followed by (iii) deprotection of the primary amine. Purification of the first intermediate, tert-butyl (3-(dimethylamino)propyl)carbamate by column chromatography (in spite of reasonably clean ${ }^{1} \mathrm{H}$ and ${ }^{13} \mathrm{C}$ NMR spectra of the crude product), was found to facilitate the isolation of the desired amine3-4 reagent in high purity without the need for time-consuming recrystallization, Fig. 6. Showing a similar trend as monomers MA2-3 and MA2-4, this amine-functional SBB reagent was somewhat less soluble in PC than the SPB ana-

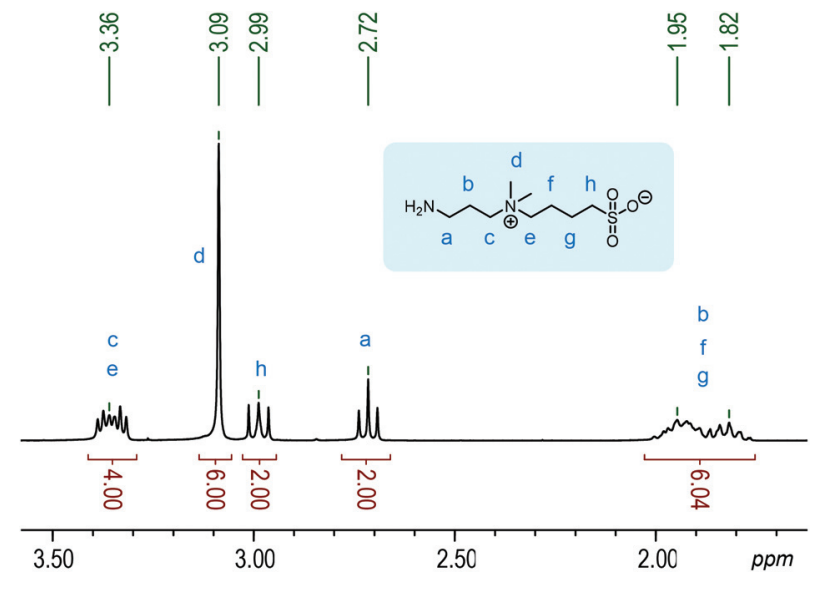

Fig. $6{ }^{1} \mathrm{H}$ NMR spectrum of sulfobutylbetaine amine recorded in $\mathrm{D}_{2} \mathrm{O}$.

logue necessitating heating in order to dissolve. Notably, however, unlike pPFPA, the generally less reactive ${ }^{59,60}$ methacrylate sister precursor poly(pentafluorophenyl methacrylate) was found to be poorly soluble in (hot) PC. This method toward zwitterionic (co)polymers thus primarily gives straightforward access to acrylamido betaine derivatives.

RAFT-prepared pPFPA with a DP of 178 and $\emptyset_{M}=1.23$ was used as the activated precursor for all modifications. Aside from requiring more heat for initial dissolution of the small molecule reagent, the synthesis of zwitterionic homo- and copolymers from pPFPA with amine3-4 did not differ from the previously described protocol ${ }^{43}$ and details of the synthesis and characterization are only briefly discussed here. A 1.5-fold excess of (a mixture of) amines dissolved in PC was added to a PC solution of pPFPA and the mixture was stirred overnight. Full substitution was confirmed by ${ }^{19} \mathrm{~F}$ NMR analysis (Fig. S2 $\dagger$ ) before product (co)polymers were purified by dialysis and isolated. In addition to a $\mathbf{p}(\mathbf{A m} \mathbf{3}-\mathbf{3})$ reference homopolymer and its SBB homologue p(Am3-4), a series of copolymers containing benzylacrylamide units, $\mathbf{p}\left[(\mathbf{A m} 3-\mathbf{4})_{\boldsymbol{x}}\right.$-co-BzAm $\left.\boldsymbol{y}\right]$, with varying molar compositions was prepared by employing mixtures of amine3-4 and benzylamine, see Table 3. Characterization by ${ }^{1} \mathrm{H}$ NMR (exemplary spectrum of $\mathbf{p}(\mathbf{A m} 3-4)$ shown in Fig. 1F) and FT-IR spectroscopy (Fig. 7) conformed to the

Table 3 Homo- and copolymers derived from pPFPA (DP $=178, \bigoplus_{M}=M_{w} / M_{n}=1.23$ ) with zwitterionic amines and benzylamine (BzA)

\begin{tabular}{|c|c|c|c|c|c|c|c|}
\hline $\begin{array}{l}\text { Product, copolymer } \\
\text { composition }\end{array}$ & $\mathrm{DP}$ & $\begin{array}{l}\text { Amine } 1 \\
\text { (feed, mol\%) }\end{array}$ & $\begin{array}{l}\text { Amine } 2 \\
\text { (feed, mol\%) }\end{array}$ & $\begin{array}{l}M_{\mathrm{n}}^{\text {theor }} \\
\left(\mathrm{kg} \mathrm{mol}^{-1}\right)\end{array}$ & 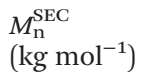 & $\bigoplus_{\mathrm{M}}^{\mathrm{SEC}}$ & $\begin{array}{l}\text { Cloud point } \\
\left({ }^{\circ} \mathrm{C}\right)\end{array}$ \\
\hline p(Am3-3) & 178 & amine3-3 (100) & - & 49.8 & 30 & 1.32 & 13.3 \\
\hline $\mathrm{p}(\mathrm{Am} 3-4)$ & 178 & amine3-4 (100) & - & 52.3 & 31 & 1.29 & 58.8 \\
\hline $\mathrm{p}\left[(\operatorname{Am} 3-4)_{0.50}-\operatorname{co}-\mathrm{BzAm}_{0.50}\right]^{a}$ & 178 & amine $3-4(50)$ & BzA (50) & 40.6 & n.d. ${ }^{b}$ & n.d. & $\mathrm{NaCl}(\mathrm{aq}){ }^{c}$ \\
\hline $\mathrm{p}\left[(\operatorname{Am} 3-4)_{0.38}-\operatorname{co}-\mathrm{BzAm}_{0.62}\right]$ & 178 & amine3-4 (35) & $\mathrm{BzA}(65)$ & 37.8 & n.d. & n.d. & $\mathrm{NaCl}(\mathrm{aq}) .^{c}$ \\
\hline $\mathrm{p}\left[(\mathrm{Am} 3-4)_{0.31}-c o-\mathrm{BzAm}_{0.69}\right]$ & 178 & amine3-4 (25) & $\operatorname{BzA}(75)$ & 36.2 & n.d. & n.d. & $\mathrm{I}^{d}$ \\
\hline
\end{tabular}

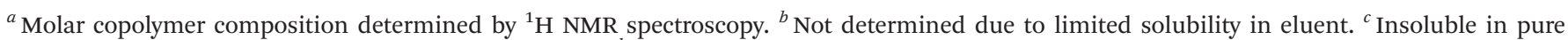

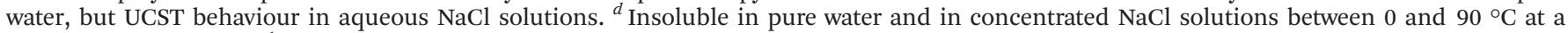
concentration of $10 \mathrm{~g} \mathrm{~L}^{-1}$. 


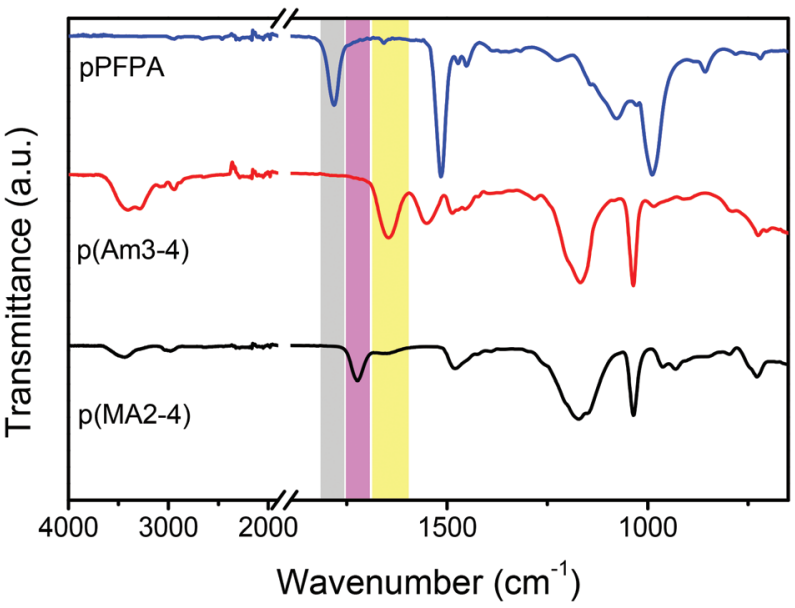

Fig. 7 FT-IR spectra of the pPFPA precursor (top), a p(Am3-4) homopolymer prepared thereof (middle), and a RAFT-made $\mathrm{p}(\mathrm{MA2}-4)$ homopolymer for comparison (bottom). The characteristic bands of the activated ester $\mathrm{C}=\mathrm{O}$ stretching $\left(1780 \mathrm{~cm}^{-1}\right.$, grey $)$, the ester $\mathrm{C}=\mathrm{O}$ stretching $\left(1720 \mathrm{~cm}^{-1}\right.$, pink), and the amide $\mathrm{C}=\mathrm{O}$ stretching $\left(1650 \mathrm{~cm}^{-1}\right.$, yellow) are marked.

expected zwitterionic (co)polymer structures. Aqueous SEC of p(Am3-3) and p(Am3-4), shown in Fig. 2C, revealed size distributions predictably similar to their pPFPA parent with both zwitterionic daughter homopolymers having very similar hydrodynamic sizes in the $0.2 \mathrm{M}$ aq. $\mathrm{NaCl}$ eluent. Due to low solubility, the benzylacrylamide (BzAM) copolymers were not characterized by SEC.

The SBB homopolymer p(Am3-4) showed a sharp, reproducible phase transition with a hysteresis of $\sim 3{ }^{\circ} \mathrm{C}$, similar to that of RAFT-made poly(meth)acrylates, see Fig. S1. $\dagger$ With a cloud point of $58.8^{\circ} \mathrm{C}$, this species had a considerably higher phase transition temperature than $\mathbf{p}(\mathbf{A m} 3-3)$ (cloud point $13.3^{\circ} \mathrm{C}$ ) suggesting again an advantage of this SBB species with regards to achieving (tuneable) UCST behaviour. Comparing samples with similar DPs (178-191) of all five zwitterionic species investigated here, we find increasing cloud points in the order: $\mathbf{p}(\mathbf{A m} 3-3) \approx \mathbf{p}(\mathbf{M A 2}-3) \ll \mathbf{p}(\mathbf{A} 2-4) \approx \mathbf{p}(\mathbf{A m} 3-4) \ll \mathbf{p}$ (MA2-4). Cloud points found here for $\mathbf{p}(\mathbf{M A 2}-3)$ were in general agreement with recently reported values of similar homopolymers ${ }^{35}$ while we note the similarity between the cloud points of $\mathbf{p}(\mathbf{A m 3}-3)\left(\mathrm{DP}=178, \mathrm{CP}=13.3^{\circ} \mathrm{C}\right)$ and $\mathbf{p}(\mathrm{MAm} 3-3)$ (reported by others, ${ }^{31} \mathrm{DP}=170, \mathrm{CP}=11.0^{\circ} \mathrm{C}$ ) suggesting a less prominent effect of the backbone methyl group in this case and/or an effect of the synthetic method.

Aside from increasing the ion bridge from a propyl to a butyl spacer, the introduction of benzylacrylamide comonomer units provides an additional handle to increase UCST transitions and the maximum salt concentration at which UCST behaviour can be observed. The cloud point dependency of the SPB copolymer $\mathbf{p}\left[(\mathbf{A m} 3-3)_{\mathbf{0 . 4 7}}-\boldsymbol{c o}-\mathbf{B z A m}_{\mathbf{0 . 5 3}}\right]$ as a function of $\mathrm{NaCl}$ concentration, taken from the literature, ${ }^{43}$ is plotted in Fig. $8 \mathrm{~A}$, indicating full solubility at $[\mathrm{NaCl}]>\sim 80 \mathrm{mM}$. Here, a series of SBB copolymers with molar BzAm contents of $50 \%$, $62 \%$, and $69 \%$, as determined by ${ }^{1} \mathrm{H}$ NMR spectroscopy in $500 \mathrm{mM} \mathrm{NaCl}$ in $\mathrm{D}_{2} \mathrm{O}$, was prepared. Notably, sample $\mathrm{p}$ [(Am34) $)_{0.31}-\boldsymbol{c o}$ - BzAm $\left._{0.69}\right]$ with the highest BzAm content did not form a clear solution in this solvent (nor at higher $\mathrm{NaCl}$ concentrations) - the determined composition may thus be less accurate-indicating an upper limit of observable zwitterionic behaviour for hydrophobic modification. The remaining two samples, however, while being insoluble in pure (hot) water, did exhibit sharp, reversible UCST transitions between clear solutions and cloudy mixtures in aqueous $\mathrm{NaCl}$ solution. As expected, the phase behaviour of these samples extended to higher $\mathrm{NaCl}$ concentrations than for the SPB copolymer, with UCST transitions measured on solutions containing up to $128 \mathrm{mM}\left(7.5 \mathrm{~g} \mathrm{~L}^{-1}\right) \mathrm{NaCl}$ for $\mathbf{p}\left[(\mathbf{A m} 3-\mathbf{4})_{\mathbf{0 . 5 0}} \boldsymbol{c o s}^{\left.-\mathbf{C z z A m}_{\mathbf{0 . 5 0}}\right]}\right.$ and $154 \mathrm{mM}\left(9.0 \mathrm{~g} \mathrm{~L}^{-1}\right.$, concentration of isotonic saline) $\mathrm{NaCl}$ for p[(Am3-4) $)_{\mathbf{0 . 3 8}}$ - $\boldsymbol{c o}$-BzAm $\left.\mathbf{0 . 6 2}_{\mathbf{6}}\right]$, see Fig. 8. These results demon-
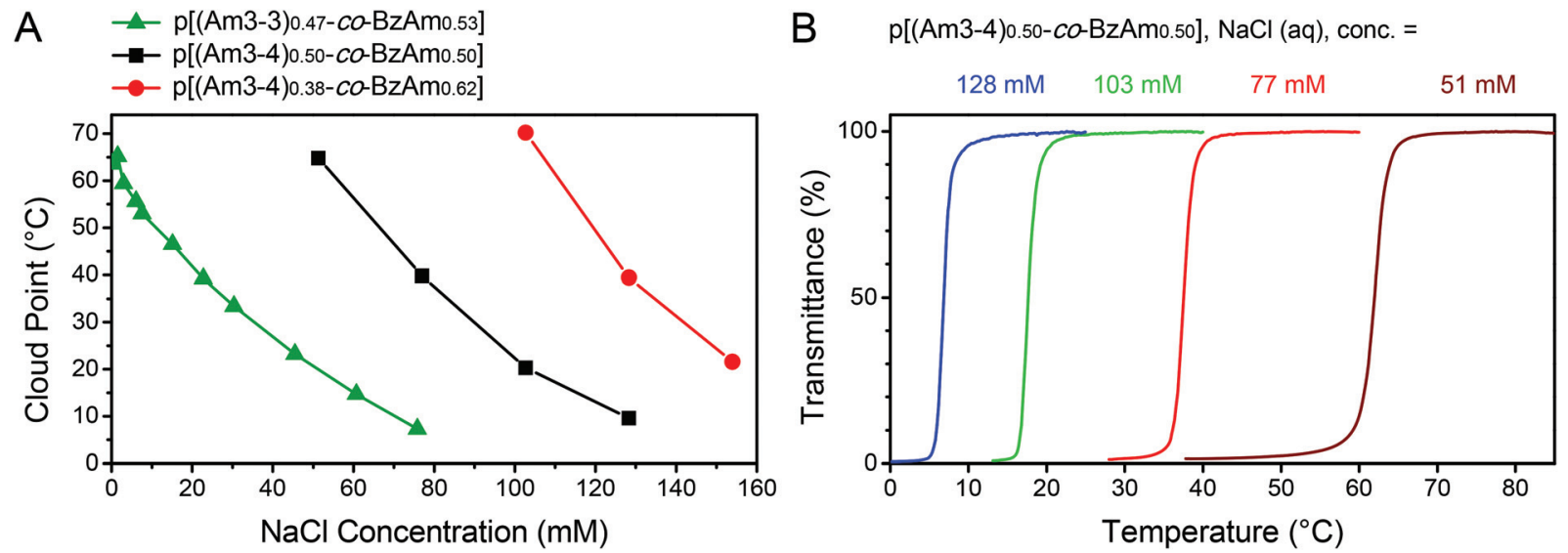

Fig. 8 Influence of $\mathrm{NaCl}$ concentration on the phase separation temperature of p(Am3-4)-benzylacrylamide copolymers; (A) plot of UCST cloud point versus $\mathrm{NaCl}$ concentration for $\mathrm{p}\left[(\mathrm{Am} 3-3)_{0.47}-\mathrm{CO}_{-}-\mathrm{BzAm}_{0.53}\right]$ (values taken from our previous study) ${ }^{43}$ (green triangles), and the $\mathrm{SBB}$ species prepared here $\mathrm{p}\left[(\mathrm{Am} 3-4)_{0.50}-\mathrm{co}-\mathrm{BzAm} \mathrm{m}_{0.50}\right]$ (black squares) and $\mathrm{p}\left[(\mathrm{Am} 3-4)_{0.38}\right.$-co-BzAmo.62] (red circles); (B) exemplary turbidity curves for p[(Am34) $\left.)_{0.50}-\mathrm{co}-\mathrm{BzAm}_{0.50}\right]$ at different $\mathrm{NaCl}$ concentrations. 
strate that the combination of SBB side chains with hydrophobic modification further increases the temperature/salt range in which sharp UCST transitions can be achieved. Specifically, UCST transitions of zwitterionic copolymers at a physiological $\mathrm{NaCl}$ concentration are promising for the exploitation of such smart materials in biomedical applications. However, the complex composition of biological fluids and the strong dependence of the UCST phase behaviour on the type of ions ${ }^{20,21,23,31}$ or, likely, other solutes needs to be considered. In the present case, for example, $\mathbf{p}\left[(\mathbf{A m} \mathbf{3}-\mathbf{4})_{\mathbf{0 . 3 8}} \mathbf{- c o}-\mathbf{B z A m}_{\mathbf{0 . 6 2}}\right]$ was found to remain soluble in phosphate buffered saline $(137 \mathrm{mM} \mathrm{NaCl}$,

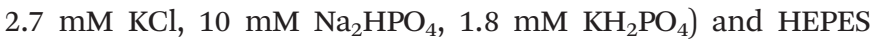
buffer (150 mM NaCl, $10 \mathrm{mM}$ 4-(2-hydroxyethyl)-1-piperazineethanesulfonic acid) when cooled to $0{ }^{\circ} \mathrm{C}$. Conversely, this strong dependence of the phase behaviour on potentially minute changes of the environment may be exploited for the development of materials that respond selectively to specific biological microenvironments with (slightly) different ion concentrations.

\section{Conclusion}

A detailed comparative study of the aqueous solution behaviour of sulfobutylbetaine (SBB) (co)polymers was presented. In all cases, samples showed significantly higher UCST transitions, i.e. they were less soluble in water, than their respective sulfopropylbetaine (SPB) counterparts. Whereas the UCST transitions of the common SPB homopolymers p(MA2-3) and p(MAm3-3) are limited to relatively high molecular weight samples and/or to solutions in ultrapure water, the corresponding SBB-functional homopolymers investigated here offer a much larger temperature and salt concentration range for observing sharp, reproducible UCST transitions. Scope and limitations of three different synthetic techniques toward SBB homo- and copolymers were compared. The quaternization of pDMAEMA with 1,4-butanesultone in 2,2,2-trifluoroethanol (TFE) suffered from low reactivity of this alkylating agent resulting in incomplete modification making this procedure less attractive for the synthesis of pristine SBB species. RAFT radical polymerization of SBB-functional (meth)acrylate monomers and their copolymerization with SPB monomers in TFE proceeded smoothly with high conversions, low measured dispersities $\emptyset_{\mathrm{M}}$, and facilitated access to a series of (co)polymers with cloud points from $13.2-76.5^{\circ} \mathrm{C}$. Low compatibility of SBBfunctional monomers with non-protic solvents including propylene carbonate (PC), however, limits the incorporation of non-polar or alcohol/water-sensitive components in this method. Post-modification of poly(pentafluorophenyl acrylate) with a SBB-functional amine in PC provided zwitterionic polyacrylamides and allowed for the introduction of hydrophobic comonomer units, but required the multi-step synthesis of the amine reagent. A SBB-functional copolymer containing $62 \mathrm{~mol} \%$ of benzylacrylamide comonomer units showed UCST behaviour up to a physiological concentration of $9 \mathrm{~g} \mathrm{~L}^{-1} \mathrm{NaCl}$ making such materials promising for exploiting their smart behaviour in the biomedical arena.

\section{Funding sources}

P. J. R. acknowledges funding from the University of New South Wales (UNSW) and the Australian Research Council (ARC) through a Discovery Early Career Researcher Award (DE120101547).

\section{References}

1 M. A. C. Stuart, W. T. S. Huck, J. Genzer, M. Muller, C. Ober, M. Stamm, G. B. Sukhorukov, I. Szleifer, V. V. Tsukruk, M. Urban, F. Winnik, S. Zauscher, I. Luzinov and S. Minko, Nat. Mater., 2010, 9, 101-113.

2 J. Seuring and S. Agarwal, Macromol. Rapid Commun., 2012, 33, 1898-1920.

3 S. Kudaibergenov, W. Jaeger and A. Laschewsky, in Supramolecular Polymers Polymeric Betains Oligomers, Springer, Berlin/Heidelberg, 2006, vol. 201, pp. 157-224.

4 A. B. Lowe and C. L. McCormick, Chem. Rev., 2002, 102, 4177-4190.

5 F. Xuan and J. Liu, Polym. Int., 2009, 58, 1350-1361.

6 A. Laschewsky, Polymers, 2014, 6, 1544-1601.

7 G. Gunkel and W. T. S. Huck, J. Am. Chem. Soc., 2013, 135, 7047-7052.

8 J. Wu, W. Lin, Z. Wang, S. Chen and Y. Chang, Langmuir, 2012, 28, 7436-7441.

9 Q. Sun, Y. Su, X. Ma, Y. Wang and Z. Jiang, J. Membr. Sci., 2006, 285, 299-305.

10 Y. Chang, Y.-J. Shih, C.-J. Lai, H.-H. Kung and S. Jiang, Adv. Funct. Mater., 2013, 23, 1100-1110.

11 J. Yuan, X. Huang, P. Li, L. Li and J. Shen, Polym. Chem., 2013, 4, 5074-5085.

12 R. Lalani and L. Liu, Biomacromolecules, 2012, 13, 1853-1863.

13 R. Hart and D. Timmerman, J. Polym. Sci., 1958, 28, 638640.

14 P. Köberle, A. Laschewsky and D. van den Boogaard, Polymer, 1992, 33, 4029-4039.

15 P. Koeberle and A. Laschewsky, Macromolecules, 1994, 27, 2165-2173.

16 V. M. Monroy Soto and J. C. Galin, Polymer, 1984, 25, 121128.

17 J. C. Salamone, W. Volksen, S. C. Israel, A. P. Olson and D. C. Raia, Polymer, 1977, 18, 1058-1062.

18 D. Kratzer, L. Barner, C. Friedmann, S. Bräse and J. Lahann, Eur. J. Org. Chem., 2014, 8064-8071.

19 M. B. Huglin and M. A. Radwan, Polym. Int., 1991, 26, 97-104.

20 P. Köberle, A. Laschewsky and T. D. Lomax, Die Makromolekulare Chemie, Rapid Commun., 1991, 12, 427-433.

21 P. Mary, D. D. Bendejacq, M.-P. Labeau and P. Dupuis, J. Phys. Chem. B, 2007, 111, 7767-7777.

22 J. Ning, G. Li and K. Haraguchi, Macromolecules, 2013, 46, 5317-5328.

23 D. N. Schulz, D. G. Peiffer, P. K. Agarwal, J. Larabee, J. J. Kaladas, L. Soni, B. Handwerker and R. T. Garner, Polymer, 1986, 27, 1734-1742. 
24 V. A. Vasantha, S. Jana, A. Parthiban and J. G. Vancso, Chem. Commun., 2014, 50, 46-48.

25 A. Bieglé, A. Mathis and J.-C. Galin, Macromol. Chem. Phys., 2000, 201, 113-125.

26 R. G. Laughlin, Langmuir, 1991, 7, 842-847.

27 J. L. Bredas, R. R. Chance and R. Silbey, Macromolecules, 1988, 21, 1633-1639.

28 Y. Chevalier and P. Le Perchec, J. Phys. Chem., 1990, 94, 1768-1774.

29 M. Galin, A. Chapoton and J.-C. Galin, J. Chem. Soc., Perkin Trans. 2, 1993, 545-553.

30 L. Chen, Y. Honma, T. Mizutani, D. J. Liaw, J. P. Gong and Y. Osada, Polymer, 2000, 41, 141-147.

31 V. Hildebrand, A. Laschewsky and D. Zehm, J. Biomater. Sci., Polym. Ed., 2014, 25, 1602-1618.

32 V. M. Monroy Soto and J. C. Galin, Polymer, 1984, 25, 254262.

33 Y.-J. Shih, Y. Chang, A. Deratani and D. Quemener, Biomacromolecules, 2012, 13, 2849-2858.

34 M. Tian, J. Wang, E. Zhang, J. Li, C. Duan and F. Yao, Langmuir, 2013, 29, 8076-8085.

35 H. Willcock, A. Lu, C. F. Hansell, E. Chapman, I. R. Collins and R. K. O’Reilly, Polym. Chem., 2014, 5, 1023-1030.

36 M. Arotcarena, B. Heise, S. Ishaya and A. Laschewsky, J. Am. Chem. Soc., 2002, 124, 3787-3793.

37 Y. Pei, J. Travas-Sejdic and D. E. Williams, Langmuir, 2012, 28, 8072-8083.

38 M. S. Donovan, B. S. Sumerlin, A. B. Lowe and C. L. McCormick, Macromolecules, 2002, 35, 8663-8666.

39 V. Strehmel, H. Wetzel, A. Laschewsky, E. Moldenhauer and T. Klein, Polym. Adv. Technol., 2008, 19, 1383-1390.

40 A. B. Lowe, N. C. Billingham and S. P. Armes, Chem. Commun., 1996, 1555-1556.

41 A. B. Lowe, N. C. Billingham and S. P. Armes, Macromolecules, 1999, 32, 2141-2148.

42 J. V. M. Weaver, S. P. Armes and V. Butun, Chem. Commun., 2002, 2122-2123.

43 P. A. Woodfield, Y. C. Zhu, Y. W. Pei and P. J. Roth, Macromolecules, 2014, 47, 750-762.
44 R. Kakuchi and P. Theato, in Functional Polymers by PostPolymerization Modification, Wiley-VCH Verlag GmbH \& Co. KGaA, 2012, pp. 45-64.

45 J. Y. Quek, P. J. Roth, R. A. Evans, T. P. Davis and A. B. Lowe, J. Polym. Sci., Part A: Polym. Chem., 2013, 51, 394-404.

46 P. J. Roth, K. T. Wiss, R. Zentel and P. Theato, Macromolecules, 2008, 41, 8513-8519.

47 R. Nudelman, O. Ardon, Y. Hadar, Y. Chen, J. Libman and A. Shanzer, J. Med. Chem., 1998, 41, 1671-1678.

48 P. J. Roth, J. Y. Quek, Y. Zhu, B. M. Blunden and A. B. Lowe, Chem. Commun., 2014, 50, 9561-9564.

49 M. Gauthier, T. Carrozzella and A. Penlidis, J. Polym. Sci., Part A: Polym. Chem., 2002, 40, 511-523.

50 P. J. Roth, C. Boyer, A. B. Lowe and T. P. Davis, Macromol. Rapid Commun., 2011, 32, 1123-1143.

51 P. van de Wetering, N. J. Zuidam, M. J. van Steenbergen, O. A. G. J. van der Houwen, W. J. M. Underberg and W. E. Hennink, Macromolecules, 1998, 31, 8063-8068.

52 H.-Y. Tian, J.-J. Yan, D. Wang, C. Gu, Y.-Z. You and X.-S. Chen, Macromol. Rapid Commun., 2011, 32, 660-664.

53 P. G. Faulkner, A. J. I. Ward and D. W. Osborne, Langmuir, 1989, 5, 924-926.

54 J. G. Weers, J. F. Rathman, F. U. Axe, C. A. Crichlow, L. D. Foland, D. R. Scheuing, R. J. Wiersema and A. G. Zielske, Langmuir, 1991, 7, 854-867.

55 W. Ding, C. Lv, Y. Sun, X. Liu, T. Yu, G. Qu and H. Luan, J. Polym. Sci., Part A: Polym. Chem., 2011, 49, 432-440.

56 G. Moad, E. Rizzardo and S. H. Thang, Aust. J. Chem., 2012, 65, 985-1076.

57 K. Skrabania, A. Miasnikova, A. M. Bivigou-Koumba, D. Zehm and A. Laschewsky, Polym. Chem., 2011, 2, 20742083.

58 P. J. Roth, F. D. Jochum, F. R. Forst, R. Zentel and P. Theato, Macromolecules, 2010, 43, 4638-4645.

59 G. B. H. Chua, P. J. Roth, H. T. T. Duong, T. P. Davis and A. B. Lowe, Macromolecules, 2012, 45, 1362-1374.

60 M. I. Gibson, E. Frohlich and H.-A. Klok, J. Polym. Sci., Part A: Polym. Chem., 2009, 47, 4332-4345. 